\title{
Computational analysis of CFSE proliferation assay
}

Tatyana Luzyanina, Sonja Mrusek, John T Edwards, Dirk Roose, Stephan Ehl, Gennady Bocharov

Report TW 463, June 2006

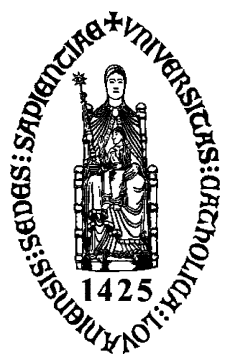

\section{Katholieke Universiteit Leuven} Department of Computer Science 


\title{
Computational analysis of CFSE proliferation assay
}

\author{
Tatyana Luzyanina ${ }^{a}$, Sonja Mrusek ${ }^{b}$, John T Edwards $^{c}$, \\ Dirk Roose $^{d}$, Stephan $\mathrm{Ehl}^{b}$, Gennady Bocharov ${ }^{e, *}$
}

\author{
${ }^{a}$ Institute of Mathematical Problems in Biology, RAS, Puschino, Russia \\ ${ }^{b}$ Children's Hospital, University of Freiburg, Germany \\ ${ }^{c}$ Mathematics Department, University of Chester, UK \\ ${ }^{d}$ Computer Science Department, Katholieke Universiteit Leuven, Belgium \\ ${ }^{e}$ Institute of Numerical Mathematics, RAS, Moscow, Russia
}

\begin{abstract}
CFSE based tracking of the lymphocyte proliferation using flow cytometry is a powerful experimental technique in immunology allowing for the tracing of labelled cell populations over time in terms of the number of divisions cells undergone. Interpretation and understanding of such population data can be greatly improved through the use of mathematical modelling. We apply a heterogeneous linear compartmental model, described by a system of ordinary differential equations similar to those proposed by Kendall. This model allows division number-dependent rates of cell proliferation and death and describes the rate of changes in the numbers of cells having undergone $j$ divisions. The experimental data set that we specifically analyze specifies the following characteristics of the kinetics of PHA-induced human T lymphocyte proliferation assay in vitro: $(i)$ the total number of live cells, $(i i)$ the total number of dead but not disintegrated cells and (iii) the number of cells divided $j$ times. Following the maximum likelihood approach for data fitting, we estimate the model parameters which, in particular, present the CTL birth- and death rate "functions". It is the first study of CFSE labelling data which convincingly shows that the lymphocyte proliferation and death both in vitro and in vivo are division number dependent. For the first time, the confidence in the estimated parameter values is analyzed by comparing three major methods: the technique based on the variance-covariance matrix, the profile-likelihood-based approach and the bootstrap technique. We compare results and performance of these methods with respect to their robustness and computational cost. We show that for evaluating mathematical models of differing complexity the information-theoretic approach, based upon indicators measuring the information loss for a particular model (Kullback-Leibler information), provides a consistent basis. We specifically discuss methodological and computational difficulties in parameter identification with CFSE data, e.g., the loss of confidence in the parameter estimates starting around the 6 -th division. Overall, our study suggests that the heterogeneity inherent in cell kinetics should be explicitly incorporated into the structure of mathematical models.
\end{abstract}

\footnotetext{
${ }^{*}$ Corresponding author
} 
Keywords: Modelling T-cell kinetics, CFSE assay, Heterogenous compartmental model, Parameter estimation, Computation of confidence intervals.

Mathematics Subject Classification (2000): 92B05, 92-08, 92C37, 92C45.

\section{Introduction}

A standard assay for the functional analysis of human $\mathrm{T}$ cells is to test their proliferative capacity to in vitro stimulation with mitogens, superantigens or conventional antigens. The tests based upon mitogens, such as $\mathrm{PHA}^{1}$ or $\mathrm{CON} \mathrm{A}^{2}$, act on naive and memory $\mathrm{T}$ lymphocytes, CD4+ and CD8+ T cells. These activators induce proliferation without the need for $\mathrm{T}$ cell receptor signalling. The tests are therefore a global assessment of the ability of $\mathrm{T}$ cells to divide and proliferate.

One conventional method that can be used to assess proliferation of cells is a $3 \mathrm{H}-$ thymidin incorporation assay. Radioactive thymidin is incorporated into the DNA of dividing cells, and the amount of radioactivity in the cell fraction is quantified. The level of radioactivity after stimulation of $\mathrm{T}$ cells with antigen versus with medium only gives an estimate of the proliferative response of the lymphocytes. A more recent FACS ${ }^{3}$ based approach to study proliferative responses has the following advantages: the avoidance of radioactivity, the ability to differentiate between CD4 and CD8 T cell responses and the ability to study the acquisition of differentiation markers during proliferation.

The flow cytometry analysis of $\mathrm{CFSE}^{4}$ labelled T-lymphocyte proliferation is a powerful technique for quantifying the labelled cell populations over time in terms of the number of divisions cells undergone. The proliferation of CFSE labelled cells is detected as progressive halving of cellular fluorescence with every cell division completed. CFSE histograms give information on the fraction of lymphocytes that have divided once, twice, etc. The percentage of T-cells having undergone from 1 up to 10 divisions can be quantified by the flow cytometry. However, giving the percentages of divided cells provides little insight into the turnover of the cells. Another factor is that the fraction of the antigen/mitogenresponsive $\mathrm{T}$ cells among the $\mathrm{PBMC}^{5}$ population used in the assay is highly variable. The advantage of the CFSE assay compared to thymidine incorporation assays is that individual responsive cells rather than bulk populations can be studied. This is particularly useful in patients with immunodeficiencies, where the numbers of T-lymphocytes might be reduced by a factor 10 to 30 . For practical purposes, it is important to describe the information on CFSE assay, related to the immune status of the patient under examination, using meaningful and simple terms, such as the responder cell frequency, the proliferation capacity of the T-lymphocyte population, the mean doubling time and its variability. A fundamental issue which still remains unexplored is whether the lymphocyte division and

\footnotetext{
${ }^{1}$ phytohemagglutinin: A phytomitogen from plants that agglutinates red blood cells. The term is commonly used specifically for the lectin obtained from the red kidney bean (Phaseolus vulgaris) which is also a mitogen that stimulates $\mathrm{T}$ lymphocytes more vigorously than B lymphocytes

${ }^{2}$ Concanavalin A: a lectin isolated from the jack bean, Canavalia ensiformis

${ }^{3}$ fluorescence activated cell sorter

${ }^{4}$ carboxyfluorescein diacetate succinimidyl ester

${ }^{5}$ peripheral blood mononuclear cells
} 
death rates depend on the number of divisions lymphocytes have undergone. A thorough quantitative interpretation and understanding of such complex population data requires the use of computational analysis based upon appropriate mathematical models.

The earlier approaches to the CFSE data analysis were based upon assumption that there is no death in the proliferating lymphocytes. The histogram data were interpreted using a simple discrete doubling model $[11,19]$ or the exponential growth model [1]. These models allowed to estimate the frequency of responding cells and the specific exponential growth rates, respectively. Later on, incorporation of the exponential death into the CFSE data analysis based upon the discrete doubling model was implemented [10]. The so called 'cellular calculus' framework was used to estimate the mean division number with its standard deviation and the average division cycle time. Recently, a systematic analysis of the parameters amenable to estimation from CFSE data was presented [9]. The biological Smith-Martin model of the cell cycle [16] was translated into a set of differential equations, modelling the structured cell division. The underlying assumption in the analysis was that the cell population is a homogenous one (i.e. the parameters of cell division and death are independent of time and the number of divisions cells have undergone).

Few aspects of T-cell heterogeneity were addressed using mathematical models. A discrete Leslie-type model was applied to estimate from CFSE data the human naive versus memory $\mathrm{T}$ cell turnover parameters: the average transit time (lag time) from division 0 to 1 , the average division rate and loss rate in all other divisions [17]. Later study [6] showed that a homogenous model of $\mathrm{T}$ lymphocyte growth is not consistent with the CFSE labelling data given in [10]. The authors considered delay differential equations for modelling the Smith-Martin scheme of cell growth. They assumed a difference in the commitment and death rates between the populations of naive lymphocytes and those which have made at least one division. The model fits well to the data representing a short-term CFSE-labelled $\mathrm{T}$ cell dynamics (hours 60 to 84 ) and predicts a substantial difference in the time needed to complete the first division and the subsequent rounds of divisions. The analysis presented in $[6,9]$ assumes that after initial transient, the lymphocyte population approaches the phase of the so called 'steady state of exponential growth'. This phase is characterized by time-independent age distribution. The validity of this simplifying assumption, which can, in general, be expected to hold true after a long time [15], in the context of the CFSE assay deserves further examination.

In the present study we address the following practically relevant issues which have not been thoroughly considered yet:

- can the lymphocyte division and death rates be reliably quantified from typical data sets on CFSE labelling using mathematical models, and are the rates dependent on the number of divisions the cells have undergone?

- reliability and performance of major computational approaches to the analysis of the confidence in the parameter estimation with respect to CFSE data fitting;

- what type of difficulties (methodological and computational) are inherent in the analysis of CFSE proliferation assay and how one could overcome those? 
The content of this paper is as follows. Using a set of original data on in vitro growth of human CFSE-labelled cells, described in Section 2, we investigate the appropriateness of a heterogeneous linear compartmental model, introduced in Section 3, for the analysis of the CFSE proliferation assay. Our kinetic analysis of the CFSE assay data is based upon the maximum likelihood parameter estimation and facilities of the MATLAB software package we use, as presented in Section 4. We show that the birth rate as a function of the division number appears to be bell-shaped, whereas the death rates are close to zero until the division age gets equal to three and increase thereafter. We compare predictions of the heterogenous model with the predictions of a simplified homogenous model which assumes the birth and death rates being constant across the experiment. The maximum likelihood approach allows us to perform the confidence analysis of the best-fit parameter estimates and to evaluate the Akaike indicators measuring the information loss for a particular model. In Section 5, we compare the performance and computational cost of three major computational methods for confidence intervals estimation: the variance-covariance matrix approach, the likelihood profile method and the bootstrap approach. To understand capabilities of the heterogenous model, we compare its performance with a version of the Smith-Martin model from [9] by considering both our in vitro data and the in vivo data presented in [9], cf. Section 6. The heterogeneous model also predicts that the in vivo turnover rates of $\mathrm{T}$ lymphocytes do depend on the lymphocyte division number. Finally, we discuss the limits of a reliable birth and death rates estimation from finite time series of CFSE data.

\section{CFSE data}

The data on cell kinetics were obtained using PHA-proliferation assay as described below. Peripheral blood mononuclear cells (PBMC) were labelled with a fluorescent dye (CFSE), which penetrates and attaches to the inner side of the cell membrane. The mitogen stimulator PHA was added to the PMBC sample in vitro to induce proliferation of $\mathrm{T}$ cells present in the sample. Each time a $\mathrm{T}$ cell divides, it looses about half of its staining intensity. At regular times after the onset of PHA-stimulation, the cells are harvested, stained with antibodies to CD4 and CD8 and analyzed by flow cytometry. The CFSE histogram represents the distribution of the cells with respect to the CFSE expression level. It is split into bins, which specify the percentages of live CD4 T cells that have undergone from 0 to 7 cell divisions. The absolute number of live cells per well was determined by microscopy. In addition to the live cells, the absolute number of dead cells was defined by the trypan blue staining microscopy. We also estimated the percentage of dead cells as determined by FACS analysis of an uptake of the dye propidium iodine (PI). A sound correlation between the dead cell counts and the PI-positive cells was observed.

The PMBC sample consists of T- and B-lymphocytes, NK cells and monocytes, the relative proportion of which is unknown. During the first three days of culture there is a significant number of live cells in the well, which are not lymphocytes. This implies that the dead cell data, at least initially, characterize the death of heterogeneous cell population, rather then the T lymphocyte population. Another confounding aspect of the 
data is that the transfer of the PMB cells from physiological in vivo conditions into in vitro culture will cause a death of many cells due to the change in milieu, temperature, plastic walls, etc. Notice that the specificity of T-lymphocyte gating gets better with time, and from day 4 to day 7 the gate used covers almost all live $T$ cells as counted. The relative error of the measurements was within $20 \%$.

Table 1 presents the set of CFSE data that we analyzed. These data specify the following characteristics of the kinetics of PHA-induced cytotoxic T lymphocyte (CTL) proliferation assay in vitro from day 3 to day 7: $(i)$ the total number of live cells, $N\left(t_{i}\right)$, (ii) the total number of dead but not disintegrated cells, $D\left(t_{i}\right)$, and (iii) the number of cells divided $j$ times, $N_{j}\left(t_{i}\right), i=0,1, \ldots, 4, j=0,1, \ldots, 7$.

\begin{tabular}{|c|c|c|c|c|c|c|c|c|c|c|}
\hline \multirow[t]{2}{*}{$\begin{array}{l}\text { Time } \\
\text { days } \\
t_{i}\end{array}$} & \multirow{2}{*}{$\begin{array}{c}\text { Total } \\
\text { number of } \\
\text { live cells } \\
N\left(t_{i}\right)\end{array}$} & \multirow{2}{*}{$\begin{array}{c}\text { Total } \\
\text { number of } \\
\text { dead cells } \\
D\left(t_{i}\right)\end{array}$} & \multicolumn{8}{|c|}{$\begin{array}{l}\text { Numbers of cells w.r.t. the number of divisions }(j) \text { they undergone } \\
\qquad N_{j}\left(t_{i}\right)\end{array}$} \\
\hline & & & 0 & 1 & 2 & 3 & 4 & 5 & 6 & 7 \\
\hline 3 & & $1.6 \times 10^{4}$ & & & & & & & & 0 \\
\hline 4 & & $2.4 \times$ & & & & & & & & 25 \\
\hline 5 & $4.4 \times$ & $6.0 \times 1$ & & & 25 & & & & 6 & 440 \\
\hline 6 & $5.0 \times$ & $1.2 \times 10^{5}$ & 500 & 12150 & 241 & 550 & & 0 & 5945 & 2150 \\
\hline 7 & $5.7 \times 10^{5}$ & $1.3 \times 10^{5}$ & 13509 & 12198 & 21603 & 51927 & 140560 & 232160 & 96102 & 3420 \\
\hline
\end{tabular}

Table 1: Quantitative dynamics of human peripheral blood mononuclear cells following stimulation with PHA in vitro. At various times, CFSE profiles were obtained by flow cytometry. The total numbers of live, $N\left(t_{i}\right)$, and dead, $D\left(t_{i}\right)$, lymphocytes, and the distribution of lymphocytes with respect to the number of divisions they have undergone, $N_{j}\left(t_{i}\right), j=0,1, \ldots, 7$, were followed from day 3 to day 7 at the indicated times $t_{i}, i=0,1, \ldots, 4$.

The availability of data on $N$ and $N_{j}$ allows one to estimate the frequency of responding precursors following the approach in [19]. For the data in Table 1 and under the simplifying assumption that there is no death, the analysis suggests that by day 7 about $72 \%$ of cells have divided at least once. In addition, one can characterize the proliferative capacity, $P C(t)$, of the population at time $t$, i.e. the average number of daughter cells generated per responding precursor by $t$. We suggest the following expression:

$$
P C\left(t_{i}\right)=\frac{N\left(t_{i}\right)-N_{0}\left(t_{i}\right)}{\sum_{j=0}^{7} \frac{N_{j}\left(t_{i}\right)}{2^{j}}-N_{0}\left(t_{i}\right)}=\frac{1-f_{0}\left(t_{i}\right)}{\sum_{j=1}^{7} \frac{f_{j}\left(t_{i}\right)}{2^{j}}},
$$

where $f_{j}:=N_{j} / N, j=0,1, \ldots, 7$, is the fraction of cells divided $j$ times, i.e. distribution of cells with respect to the number of divisions they undergone. The CFSE assay data suggest, cf. Fig. 1, that by day 7 the proliferation capacity of the population reaches a peak value of 15.7 cells per responding cell with the estimated maximum expansion factor of about 3 per cell per day. The overall proliferation capacity curve follows a logistic growth pattern and the increase rate slows down after day 4 .

The above approach is useful in characterizing the net growth parameters of the temporal evolution of a cell population. In reality, cell kinetics is affected by the cellular heterogeneity with respect to the division rate, death, etc. It is important practically to 
assess more informative quantities such as generation times, the mean number of divisions, etc. Let us define mathematically the above parameters and highlight the connection between them.

The doubling time of the population, $\tau_{d}$, is defined as the time it takes population to double. It is related to the exponential growth rate ( say $\alpha$ ) by $\tau_{d}=\ln (2) / \alpha$. This quantity characterizes the excess of births over deaths in a unit time interval. The mean generation time is defined as the age $s$ at which a cell divides measured from birth of the cell. The generation time varies from cell to cell. If one denotes the generation time distribution function by $g(s)$, then the mean generation time can be determined as $\tau_{g}=\int_{0}^{\infty} s g(s) \mathrm{d} s$, whereas the variance in the generation times is defined as $\sigma_{g}^{2}=\int_{0}^{\infty}\left(\tau_{g}-s\right)^{2} g(s) \mathrm{d} s$ [15]. Although one can expect the mean doubling time to be longer than the mean generation time, in fact it appears to be the other way around. The approximate relationship is presented in [15] as $\tau_{g} \approx \tau_{d}+0.151 \sigma_{g}^{2} / \tau_{d}^{2}$.

Another useful time-dependent characteristics of cell kinetics are the growth of the total cell population $N(t)$, the numbers of precursors $P(t)$ that would have generated the current lymphocyte population in the absence of death, the mean number of divisions $\mu(t)$ and the variance $\sigma^{2}$ in the number of divisions cells have undergone, which are defined, cf., e.g. [9], as

$N(t)=\sum_{j=0}^{7} N_{j}(t), \quad P(t)=\sum_{j=0}^{7} N_{j}(t) 2^{-j}, \quad \mu(t)=\sum_{j=0}^{7} j f_{j}(t), \quad \sigma^{2}(t)=\sum_{j=0}^{7} j^{2} f_{j}(t)-\mu^{2}(t)$.

These can be easily computed either using the data or the "best-fit" solution of a mathematical model of cell kinetics, cf. Section 4.

\section{Mathematical models}

To model the population dynamics of CFSE labelled lymphocytes following the PHA stimulation, we consider two compartmental models, described by systems of linear ordinary differential equations (ODEs). Mathematically, these models are just two versions of the discrete compartmental model by Kendall [12], originally proposed for describing the age-dependent cell cycle progression. In our setting, the compartments represent the cell populations, which have made a specified number of divisions, rather than the stages of the cell cycle as considered originally. The two models, that we refer to as heterogenous and homogenous compartmental models of cell population kinetics, differ by the assumption on the dependence of lymphocytes proliferation and death rates on the number of divisions lymphocytes undergone. Below $N_{j}(t)$ and $D(t)$ denote the population sizes at time $t$ of live lymphocytes having undergone $j$ divisions and dead but not disintegrated lymphocytes, respectively.

\subsection{Heterogenous compartmental model}

The heterogenous compartmental model assumes that the per capita proliferation and death rates of T-lymphocytes, $\alpha_{j}$, respectively $\beta_{j}$, depend on the number of divisions 
lymphocytes undergone. The rates of change of $N_{j}(t)$ and $D(t)$ with time is represented by the set of equations

$$
\begin{aligned}
& \frac{d N_{0}}{d t}(t)=-\left(\alpha_{0}+\beta_{0}\right) N_{0}(t), \\
& \frac{d N_{j}}{d t}(t)=2 \alpha_{j-1} N_{j-1}(t)-\left(\alpha_{j}+\beta_{j}\right) N_{j}(t), \quad j=1, \ldots, J, \\
& \frac{d D}{d t}(t)=\sum_{j=0}^{J} \beta_{j} N_{j}(t)-\delta D(t) .
\end{aligned}
$$

The first term on the right of equations for $N_{j}(t)$ represents the cell birth (influx from previous compartment because of division), while the last term on the right represents cell loss (outflux from the compartment) due to division and death. In the equation for dead cells, $\delta$ denotes the specific (fractional) decay rate of dead lymphocytes due to disintegration and phagocytosis.

Assuming that the population sizes at time $t_{0}$ are specified by initial data $N_{j}\left(t_{0}\right)$ and $D\left(t_{0}\right)$, and the condition $\alpha_{j}+\beta_{j} \neq \alpha_{i}+\beta_{i}$ is fulfilled for $i \neq j$, the solution of the model is expressed in the form

$$
\begin{aligned}
N_{j}(t)= & \sum_{s=1}^{j}\left\{2^{s} N_{j-s}\left(t_{0}\right) \prod_{m=j-s}^{j-1} \alpha_{m} \sum_{i=j-s}^{j} \mathrm{e}^{-c_{i}\left(t-t_{0}\right)} \prod_{k=j-s, k \neq i}^{j}\left(c_{k}-c_{i}\right)^{-1}\right\}+N_{j}\left(t_{0}\right) \mathrm{e}^{-c_{j}\left(t-t_{0}\right)}, \\
& j=0,1, \ldots J, \quad t \geq t_{0}, \\
D(t)= & \sum_{j=0}^{J} \beta_{j}\left\{\sum_{s=1}^{j} 2^{s} N_{j-s}\left(t_{0}\right) \prod_{m=j-s}^{j-1} \alpha_{m} \sum_{i=j-s}^{j} \frac{\mathrm{e}^{-c_{i}\left(t-t_{0}\right)}-\mathrm{e}^{-\delta\left(t-t_{0}\right)}}{\delta-c_{i}} \prod_{k=j-s, k \neq i}^{j}\left(c_{k}-c_{i}\right)^{-1}\right\} \\
& +\sum_{j=0}^{J} \beta_{j} N_{j}\left(t_{0}\right) \frac{\mathrm{e}^{-c_{j}\left(t-t_{0}\right)}-\mathrm{e}^{-\delta\left(t-t_{0}\right)}}{\delta-c_{j}}+D\left(t_{0}\right) \mathrm{e}^{-\delta\left(t-t_{0}\right)}, \quad t \geq t_{0},
\end{aligned}
$$

where $c_{j}:=\alpha_{j}+\beta_{j}$. The availability of the closed-form solution to the model reduces the computational treatment of the model-driven data analysis.

\subsection{Homogenous compartmental model}

A simplified version of the heterogenous model can be obtained if we assume that the proliferation and death rates of cells, $\alpha$ and $\beta$, do not depend on the number of divisions cells undergone. For practical examples, we refer to cell kinetics studies $[6,9,14,15]$. The corresponding 'homogenous compartmental model', is defined by the following system of ODEs

$$
\begin{aligned}
& \frac{d N_{0}}{d t}(t)=-(\alpha+\beta) N_{0}(t), \\
& \frac{d N_{j}}{d t}(t)=2 \alpha N_{j-1}(t)-(\alpha+\beta) N_{j}(t), \quad j=1, \ldots, J, \\
& \frac{d D}{d t}(t)=\sum_{j=0}^{J} \beta N_{j}(t)-\delta D(t),
\end{aligned}
$$


with the same initial data as for heterogenous model (3.1). The solution of this model for $t \geq t_{0}$ is given by

$$
\begin{aligned}
N_{j}(t)= & \mathrm{e}^{-c\left(t-t_{0}\right)} \sum_{i=0}^{j}(2 \alpha)^{i} \frac{\left(t-t_{0}\right)^{i}}{i !} N_{j-i}\left(t_{0}\right), \quad j=0,1, \ldots, J, \\
D(t)= & \beta \sum_{j=0}^{J} \sum_{i=0}^{j}(2 \alpha)^{i} N_{j-i}\left(t_{0}\right)\left\{\frac{(-1)^{i}}{(\delta-c)^{i+1}}\left(\mathrm{e}^{(\delta-c)\left(t-t_{0}\right)} \sum_{k=i}^{0} \frac{\left(\left(t-t_{0}\right)(c-\delta)\right)^{k}}{k !}-1\right)\right\} \mathrm{e}^{-\delta\left(t-t_{0}\right)} \\
& +D\left(t_{0}\right) \mathrm{e}^{-\delta\left(t-t_{0}\right)},
\end{aligned}
$$

where $c:=\alpha+\beta$.

\section{Parameter estimation}

We search for a vector of best-fit parameters, $\mathbf{p}^{*}$, for which the model solution $N_{j}\left(t ; \mathbf{p}^{*}\right)$, $D\left(t ; \mathbf{p}^{*}\right)$ is closest, in a certain sense, to the given experimental data at the time points of the measurements, i.e. the solution fits the data in an optimal way. The vector $\mathbf{p}, \mathbf{p} \in \mathbb{R}^{L}$, has the components

$$
\mathbf{p}:=\left[\alpha_{0}, \alpha_{1}, \ldots, \alpha_{7}, \beta_{0}, \beta_{1}, \ldots, \beta_{7}, \delta\right],
$$

and

$$
\mathbf{p}:=[\alpha, \beta, \delta]
$$

for models (3.1) and (3.3), respectively. Our data set consists of cell numbers $N_{j}^{i}:=N_{j}\left(t_{i}\right)$ and $D^{i}:=D\left(t_{i}\right)$ measured at times $t_{i}$, cf. Table 1 , where $t_{0}=72, t_{1}=96, t_{2}=120, t_{3}=$ $144, t_{4}=168$ (hours). The values $N_{j}^{0}$ and $D^{0}$ are used as the initial data $N_{j}\left(t_{0}\right)$ and $D\left(t_{0}\right)$ for the models.

\subsection{Maximum likelihood approach}

To estimate the division and death rates of cells, we fit a model to the given data set by maximizing the likelihood that the data did arise from the model. Applying the maximum likelihood approach to our problem, we assume that

- the observational errors, i.e. the residuals defined as a difference between observed and model-predicted values, are normally distributed,

- the errors in observations at successive times are independent,

- the errors in the components of the state vector are independent,

- the variance of observation errors $\left(\sigma^{2}\right)$ is the same for all the state variables and observation times. 
Under these conditions, the log-likelihood function specifying the probability of observing the given data set is given by

$$
\ln (\mathcal{L}(\mathbf{p} ; \sigma))=-0.5\left(n_{d} \ln (2 \pi)+n_{d} \ln \left(\sigma^{2}\right)+\sigma^{-2} \Phi(\mathbf{p})\right),
$$

where $n_{d}$ is the total number of scalar measurements and $\Phi(\mathbf{p})$ is an ordinary least-squares function

$$
\Phi(\mathbf{p})=\sum_{i=1}^{4}\left(\sum_{j=0}^{7}\left(N_{j}^{i}-N_{j}\left(t_{i} ; \mathbf{p}\right)\right)^{2}+\left(D^{i}-D\left(t_{i} ; \mathbf{p}\right)\right)^{2}\right),
$$

see $[2,3]$ for further details.

The problem of maximizing the likelihood function is equivalent to that of minimizing $\Phi(\mathbf{p})$, provided that $\sigma^{2}$ is assigned the value

$$
\sigma^{*^{2}}=\frac{1}{n_{d}} \Phi\left(\mathbf{p}^{*}\right),
$$

which follows from the optimality condition $\partial\left(\ln \left(\mathcal{L}\left(\mathbf{p}^{*} ; \sigma\right)\right)\right) / \partial \sigma^{2}=0$. Here $\mathbf{p}^{*}$ is the parameter vector, which gives a minimum to the ordinary least-squares function.

Relevant details of the computational treatment of the minimization problem are presented in Section 5.4. Figure 2 shows the experimental data and the solutions of the two models corresponding to the best-fit parameter estimates. The kinetics of cells which have undergone more than two divisions is consistently reproduced by the heterogenous model. In contrast, the data characterizing the kinetics of the first two divisions appear to be a problem for both models. This discrepancy might be related to a large observation error for the number of $\mathrm{T}$ cells that have undergone one or two divisions. Indeed, the decline in the number of undivided cells is not accompanied by an increase in the number of cells that have divided once or twice, which seems to be counterintuitive. As shown in Fig. 3 (left), the growth of the total cell population $N$ slows down after day 5 . This concave pattern is consistently captured by the heterogenous model, whereas the homogenous model predicts a biased dynamics. The numbers of precursors $P$ estimated from the data and predicted by the models are close to each other. Figure 3 (right) shows that the mean number of divisions cell populations have undergone $(\mu)$ is predicted reliably by the heterogenous model. However, the evolution of the variance $\left(\sigma^{2}\right)$ in the mean division number over time is not precisely reproduced. The homogenous models gives a poor fit of the $\mu$ and $\sigma^{2}$.

The value of the objective function at the computed minimum is $\Phi\left(\mathbf{p}^{*}\right) \approx 1.27 \times 10^{9}$ for model (3.1) and $\Phi\left(\mathbf{p}^{*}\right) \approx 6.15 \times 10^{10}$ for model (3.3). Obviously, an increase in the number of the heterogenous model parameters provides a better description of the data in terms of the objective function. On the other hand, small data sets do not support complex models with a number of parameters above some threshold as the variance in the parameter estimates increases (see the next section). The information-theoretic approach to model evaluation suggests that in addition to the bias in the data description one has to consider the parsimony principle, i.e., to incorporate the number of observations and the number of parameters in quantitative ranking of different models [5]. The Akaike criterion, characterizing the information complexity of the models, is based upon the 
Kullback-Leibler notion of the directed distance between the given model and an 'ideal model' of the data. It makes use of the maximum likelihood estimation to evaluate the information loss associated with the specific model for the given data set. Because our interest is in the relative size of the indicators, we evaluate the revised Akaike indicators obtained by discarding extraneous terms,

$$
\begin{gathered}
\mu_{A I C}=n_{d} \ln \left(\Phi\left(\mathbf{p}^{*}\right)\right)+2(L+1), \\
\mu_{c A I C}=n_{d} \ln \left(\Phi\left(\mathbf{p}^{*}\right)\right)+2(L+1)+\frac{2(L+1)(L+2)}{n_{d}-L-2},
\end{gathered}
$$

see [5] for technical details. The first version (4.4a) is satisfactory if $n_{d}>40(L+1)$, otherwise (4.4b) should be used. The value of Akaike index $\mu_{c A I C}$ equals approximately 831 and 904 for models (3.1) and (3.3), respectively. Therefore, the heterogenous model is closer to the true model of the data as indicated by the smaller value of the information loss.

\subsection{Best-fit T-lymphocyte turnover parameters}

Parameter estimation results obtained using the ordinary least-squares approach for models (3.1) and (3.3) are summarized in Table 2. The best-fit estimates of the heterogenous model parameters suggest that the lymphocyte proliferation and death rates are not constant but vary essentially with the division number in a non-monotone way, cf. Fig. 4. Indeed, the division rate increases by about four-fold between the naive cells and the cells that have undergone two divisions and gradually declines for cells which made more than three divisions. The mean population doubling time given in Table 2 is about two days for the first division of naive lymphocytes and shortens to 13 hours after two divisions. The death rates appear to be close to zero until the division age of cells gets three and after that they start to increase. Importantly, the turnover parameter estimates look counter-intuitive for cells older than six divisions and this aspect will be analyzed below.

The estimation results obtained with the homogenous model, cf. Table 2 and Fig. 4, are different from those of the heterogenous model and, in the context of division number structured cell kinetics, do not provide appropriate characterization of the turnover. Therefore, the use of the homogenous model does not seem to be justified for parameter estimation of cell kinetics.

The best-fit parameter estimates can be used to predict the number of lymphocytes responding to PHA-stimulation, i.e. the value $N_{0}$ at time $t=0$. From the explicit solution of model (3.1), the kinetics of naive lymphocytes follows the exponential behavior

$$
N_{0}(t)=N_{0}(72) \mathrm{e}^{-\left(\alpha_{0}+\beta_{0}\right)(t-72)}, \quad t \geq 72 \text { (hours). }
$$

Backward extrapolation from 72 hours to the beginning of the experiment suggests that the number of responding lymphocytes at time $t=0$ equals $7.54 \times 10^{4}$ cells, i.e. about $38 \%$ out of the total $2 \times 10^{5}$ cells added to the culture on day 0 respond to the PHA stimulation. The accuracy of the estimate, e.g. the $95 \%$ confidence range, can be evaluated using the results of Table 3 . The corresponding confidence interval is [29\%, 54\%]. 


\begin{tabular}{|l|c|c|c|c|c|c|}
\hline $\begin{array}{l}\text { Heterogenous } \\
\text { model (3.1) }\end{array}$ & $\begin{array}{c}\text { Division rate } \\
(1 / \text { hour })\end{array}$ & $\begin{array}{c}\text { Death rate } \\
(1 / \text { hour })\end{array}$ & $\begin{array}{c}\text { Disintegration } \\
\text { rate }(1 / \text { hour })\end{array}$ & $\begin{array}{c}\text { Doubling time } \\
\text { (hours })\end{array}$ \\
\hline$\alpha_{0}$ & $1.31 \times 10^{-2}$ & $\beta_{0}$ & $2.33 \times 10^{-15}$ & $\delta$ & $4.52 \times 10^{-2}$ & 52.8 \\
$\alpha_{1}$ & $3.10 \times 10^{-2}$ & $\beta_{1}$ & $7.98 \times 10^{-13}$ & & & 22.4 \\
$\alpha_{2}$ & $5.21 \times 10^{-2}$ & $\beta_{2}$ & $5.56 \times 10^{-13}$ & & & 13.3 \\
$\alpha_{3}$ & $4.95 \times 10^{-2}$ & $\beta_{3}$ & $1.54 \times 10^{-14}$ & & & 14.0 \\
$\alpha_{4}$ & $2.94 \times 10^{-2}$ & $\beta_{4}$ & $7.12 \times 10^{-3}$ & & & 23.5 \\
$\alpha_{5}$ & $7.28 \times 10^{-3}$ & $\beta_{5}$ & $2.69 \times 10^{-2}$ & & & 95.3 \\
$\alpha_{6}$ & $2.26 \times 10^{-2}$ & $\beta_{6}$ & $7.07 \times 10^{-15}$ & & & 30.6 \\
$\alpha_{7}$ & 1.37 & $\beta_{7}$ & $6.249 \times 10^{-11}$ & & & 0.5 \\
\hline Homogenous & & & & & & \\
model (3.3) & & & & & & 32.5 \\
\hline$\alpha$ & $2.13 \times 10^{-2}$ & $\beta$ & $3.35 \times 10^{-3}$ & $\delta$ & $5 \times 10^{-18}$ & \\
\hline
\end{tabular}

Table 2: Best-fit parameter estimates of the heterogenous model (3.1) and the homogenous model (3.3) obtained using the ordinary least-squares approach and the data set in Table 1.

Sensitivity analysis allows one to rank the effect of variation in the model parameters on the division structured cell kinetics. Figure 5 shows sensitivity coefficients, $\partial N_{j}\left(t ; \mathbf{p}^{*}\right) / \partial p_{k}$, with respect to the components $\alpha_{0}, \alpha_{4}, \beta_{4}$ of the parameter vector. The left figure shows that the effect of variation in the division rate of the naive lymphocytes is amplified via a succession of proliferation events as manifested by the relative size and position of the peaks of the sensitivity curves. Indeed, the peak values become higher for cell compartments with longer proliferation history. The sensitivity functions presented in Fig. 5 (right) show the effect of variation in the birth (top) and death (bottom) rates of cells which have undergone four divisions. For example, an increase in $\alpha_{4}$ leads to a reduction in $N_{4}$ and to an increase in $N_{5}, N_{6}, N_{7}$ and $D$. An increase of the death rate $\beta_{4}$ decreases the population sizes of cells at later generations.

\subsection{Practical identifiability}

The best-fit estimates of parameters $\beta_{j}, j=0,1,2,3,6,7$, are close to zero, taking numerical values ranging between $10^{-15}$ and $10^{-11}$ (hours $^{-1}$ ). Biologically, these small values would imply zero death rate of the proliferating cells with division number age from zero to three, six and seven. The prediction of no death in cells, that have made six and more divisions, might rather reflect that the data for corresponding populations are not informative enough for reliable estimation of the death rate (see discussion below). To clarify whether setting the above parameters to zero is justified mathematically, we examined the behavior of the least-squares function $\Phi$ as a function of pairwise combinations of selected parameters. Note that visualization of the shape of the used objective function in neighborhoods of the best-fit values of the model parameters allows one to analyze the reliability of the computed best-fit. Moreover, our parameter estimation problem is a constrained optimization problem since the components of vector $\mathbf{p}$ are required to be 
non-negative. In this case, the optimal values for some of the parameters can be attained at the feasible region border. Figure 6a illustrates a plane-type behavior of $\Phi\left(\beta_{0}, \beta_{1}\right)$ so that the smallest value of $\Phi$ is reached at $\left(\beta_{0}, \beta_{1}\right)=(0,0)$. The function $\Phi\left(\alpha_{1}, \beta_{1}\right)$ with contour plots displaying valley-type behavior, cf. Fig. $6 \mathrm{~b}$, indicates that the fitting is not sensitive to the value of $\beta_{1}$ within the examined range $\left[0,1.5 \times 10^{-12}\right]$. The described features are typical for the parameters $\beta_{j}, j=0,1,2,3,6,7$. The above analysis suggests that these parameters can be set equal to zero. If we treat them as fixed ad hoc and perform the data fitting by varying the remaining 11 parameters, i.e., using the vector of estimated parameters

$$
\mathbf{p}=\left[\alpha_{0}, \alpha_{1}, \alpha_{2}, \ldots, \alpha_{7}, \beta_{4}, \beta_{5}, \delta\right],
$$

the best-fit values of the objective function and the parameters are the same (within machine accuracy) as in the case of the parameter vector with 17 components.

In contrast to the above case, (locally) elliptic contours and a 'parabolic'-type shape of $\Phi\left(\beta_{4}, \beta_{5}\right)$, cf. Fig. 6c, suggest that the parameters $\beta_{4}$ and $\beta_{5}$ are well defined. Similar behavior is observed for the rest of pairwise parameter combinations not involving the above mentioned $\beta_{j}$, e.g. Fig. 6 d. Notice that the local behavior of the objective function may not represent the 'global' one, as follows by comparing Fig. 6e and its zoomed-out version in Fig. $6 f$.

The CFSE data set presented in Table 1 does not ensure a reliable estimation of the division and death rates for cells which have undergone more than 6 divisions. Indeed, the best-fit value of $\alpha_{7}$ is very high, whereas $\beta_{6}$ and $\beta_{7}$ are close to zero. Because these parameters affect only the kinetics of cells which have made 7 divisions, the unrealistic values might be attributed to a relatively small contribution of the experimental data, characterizing the number of cells having done 7 divisions, to the objective function. This point is illustrated in Fig. 6 (bottom raw). For $\Phi\left(\alpha_{6}, \alpha_{7}\right)$, the contour plots are regular circles in the $\left(\alpha_{6}, \alpha_{7}\right)$-plane in a small neighborhood of the computed minimum, while $\Phi\left(\alpha_{6}, \alpha_{7}\right)$ does not change much when we consider a larger interval of variation of $\alpha_{7}$. The corresponding minimum of $\Phi$ can be regarded as a 'weak' one with respect to $\alpha_{7}$, implying that a large change in the parameter value results in a small change in the value of the objective function. A similar, although much less pronounced, feature is observed for $\alpha_{6}$ (not shown). This implies that the confidence in the best-fit parameter estimates deserves further examination. Assessing the uncertainties in the parameter estimates is an essential part of the quantitative characterization of $\mathrm{T}$ cell turnover.

\section{Confidence of the parameter estimation}

The general approach to characterize the reliability of parameter estimations is based upon evaluating their confidence intervals (CIs). We have performed computational analysis of the confidence intervals for the vector of 11 parameters (4.5) of the heterogenous model (3.1) with $\beta_{j}=0, j=0,1,2,3,6,7$, as fixed ad hoc. There exist three major approaches to evaluate CIs: the technique based on the variance-covariance matrix [4], the profilelikelihood-based method [18] and two variants of the bootstrap method - parametric and 
non-parametric ones [7]. We apply these methods and assess their relative performance by computing approximations to $95 \%$ confidence intervals for the estimated parameters.

In the following, $\Phi\left(\mathbf{p}^{*}\right)$ stands for the optimized least-squares function, $n_{d}$ is the number of the experimental data used and $n_{p}$ is the number of the estimated parameters. In our case, $n_{d}=36$, since the data on day 3 are used as the initial values and are, therefore, discarded from the fitting, and $n_{p}=11$.

\subsection{Variance-covariance analysis}

The variance-covariance method is based upon a parabolic approximation of the objective function around the best-fit parameter estimate $\mathbf{p}^{*}$. The $100 \cdot \theta \%$ confidence interval for the parameter of interest, e.g. for $p_{k}$, is approximated by the standard interval

$$
C I_{p_{k}}=\left[p_{k}^{*}-\sigma_{p_{k}} z\left(\theta, n_{f}\right), p_{k}^{*}+\sigma_{p_{k}} z\left(\theta, n_{f}\right)\right], \quad k=1,2, \ldots, n_{p},
$$

where $p_{k}^{*}$ is the best-fit parameter estimate, $\sigma_{p_{k}}$ is the standard deviation for $p_{k}$ and $z\left(\theta, n_{f}\right)$ is the $100 \cdot \theta$ percentage point of the Student's $t$-distribution with $n_{f}:=n_{d}-n_{p}$ degrees of freedom. An estimate of the standard deviation of $p_{k}$ is computed as follows. First we construct the covariance matrix

$$
\Xi\left(\mathbf{p}^{*}\right)=\frac{2 \Phi\left(\mathbf{p}^{*}\right)}{n_{d}-n_{p}} H^{-1}\left(\mathbf{p}^{*}\right) \in \mathbb{R}^{n_{p} \times n_{p}}
$$

where $H$ is the Hessian matrix,

$$
H(\mathbf{p}):=\left\{\frac{\partial}{\partial \mathbf{p}}\right\}\left\{\frac{\partial}{\partial \mathbf{p}}\right\}^{T} \Phi(\mathbf{p}) \in \mathbb{R}^{n_{p} \times n_{p}}, \quad H_{k, m}(\mathbf{p})=\frac{\partial^{2}}{\partial p_{k} \partial p_{m}} \Phi(\mathbf{p}),
$$

with $H_{k, m}$ being the $(k, m)$-th element of $H$. The standard deviation for the $k$-th element of $\mathbf{p}$ is given by the corresponding diagonal element $\Xi_{k, k}$ of the covariance matrix,

$$
\sigma_{k}=\sqrt{\Xi_{k, k}\left(\mathbf{p}^{*}\right)}
$$

The computed estimates of $95 \%$ CIs $(z \approx 2.06)$ for the best-fit parameters of model (3.1) are presented in Table 3 and shown in Fig. 7. The intervals appear to be quite narrow for all parameters except $\alpha_{6}$ and $\alpha_{7}$. The estimated CIs indicate that data sets covering 7 divisions $(J)$, such as presented in Table 1, are informative enough to estimate reliably the proliferation and death rates of the first six $(J-1)$ successive divisions. Note that the variance in the parameter estimates for the homogenous model is much narrower: $\mathrm{CI}_{\alpha} \approx[2.12,2.14] \times 10^{-2}$ and $\mathrm{CI}_{\beta} \approx[3.30,3.39] \times 10^{-3}$.

The variance-covariance matrix can also be used to evaluate the joint confidence regions for the elements of $\mathbf{p}^{*}$. In addition, its off-diagonal elements provide insight into the correlation between the best-fit estimates, cf. Table 4 . Interestingly, the correlation is particularly high between the turnover parameters of cells having made four and five divisions. About 50\% of the parameter combinations have quite low (below 0.2) correlation. 


\begin{tabular}{|c|c|c|c|c|c|}
\hline $\mathbf{p}$ & \multirow{2}{*}{$\begin{array}{c}\text { Best-fit } \\
\text { values }\end{array}$} & \multicolumn{4}{|c|}{ Estimates of 95\% confidence intervals } \\
\cline { 5 - 6 } & & $\begin{array}{c}\text { variance-covariance } \\
\text { method }\end{array}$ & $\begin{array}{c}\text { profile likelihood } \\
\text { method }\end{array}$ & \multicolumn{2}{|c|}{ bootstrap method } \\
\cline { 5 - 6 } & & & & non-parametric & parametric \\
\hline & & & & & \\
$\alpha_{0}$ & $1.31 \times 10^{-2}$ & {$[0.79,1.8] \times 10^{-2}$} & {$[0.94,1.8] \times 10^{-2}$} & {$[0,2.9] \times 10^{-2}$} & {$[0.51,2.1] \times 10^{-2}$} \\
$\alpha_{1}$ & $3.10 \times 10^{-2}$ & {$[2.1,4.1] \times 10^{-2}$} & {$[2.4,4.0] \times 10^{-2}$} & {$[1.1,5.1] \times 10^{-2}$} & {$[2.0,4.2] \times 10^{-2}$} \\
$\alpha_{2}$ & $5.21 \times 10^{-2}$ & {$[4.1,6.4] \times 10^{-2}$} & {$[4.4,6.3] \times 10^{-2}$} & {$[3.1,7.4] \times 10^{-2}$} & {$[3.8,6.7] \times 10^{-2}$} \\
$\alpha_{3}$ & $4.95 \times 10^{-2}$ & {$[4.2,5.7] \times 10^{-2}$} & {$[4.4,5.6] \times 10^{-2}$} & {$[3.0,6.9] \times 10^{-2}$} & {$[4.1,5.8] \times 10^{-2}$} \\
$\alpha_{4}$ & $2.94 \times 10^{-2}$ & {$[2.1,3.7] \times 10^{-2}$} & {$[2.4,3.5] \times 10^{-2}$} & {$[1.4,4.5] \times 10^{-2}$} & {$[2.3,3.6] \times 10^{-2}$} \\
$\alpha_{5}$ & $7.28 \times 10^{-3}$ & {$[0.24,1.2] \times 10^{-2}$} & {$[0.42,1.3] \times 10^{-2}$} & {$[0,2.2] \times 10^{-2}$} & {$[0.32,1.1] \times 10^{-2}$} \\
$\alpha_{6}$ & $2.26 \times 10^{-2}$ & {$[0,5.5] \times 10^{-2}$} & {$[0.09,5.8] \times 10^{-2}$} & & {$[0,4.8] \times 10^{-2}$} \\
$\alpha_{7}$ & 1.37 & {$[0,6.8]$} & {$[0.016, \infty)$} & & {$[0,2.7]$} \\
$\beta_{4}$ & $7.12 \times 10^{-3}$ & {$[0,1.8] \times 10^{-2}$} & {$[0,1.51] \times 10^{-2}$} & {$[0,2.6] \times 10^{-2}$} & {$[0,1.7] \times 10^{-2}$} \\
$\beta_{5}$ & $2.69 \times 10^{-2}$ & {$[1.2,4.2] \times 10^{-2}$} & {$[1.0,3.8] \times 10^{-2}$} & {$[0,5.6] \times 10^{-2}$} & {$[1.4,3.9] \times 10^{-2}$} \\
$\delta$ & $4.52 \times 10^{-2}$ & {$[2.7,6.4] \times 10^{-2}$} & {$[2.9,6.0] \times 10^{-2}$} & {$[0,9.4] \times 10^{-2}$} & {$[2.9,6.1] \times 10^{-2}$} \\
\hline
\end{tabular}

Table 3: Computational estimates of $95 \%$ confidence intervals for the best-fit parameter values of the heterogenous model (3.1) approximated by the variance-covariance, profile-likelihood and bootstrap methods.

The accuracy of the confidence intervals computed by the variance-covariance method depends on how consistent the local parabolic approximation of the objective function in the vicinity of the best-fit parameters is. Other methods need to be applied to assess the validity of the variance analysis.

\subsection{Profile-likelihood-based method}

Profile-likelihood method provides a method for computing the confidence intervals of the maximum likelihood parameter estimates by following 'a global' behaviour of the objective function [18]. To compute approximations to the $95 \%$ CIs of the estimates, we proceed as follows. For a parameter of interest, $p_{k}^{*}$, we search for the interval $\left[p_{k}^{\min }, p_{k}^{\max }\right]$ of maximal width and containing $p_{k}^{*}$ such that

$$
\left|\ln (\mathcal{L}(\tilde{\mathbf{p}}))-\ln \left(\mathcal{L}\left(\mathbf{p}^{*}\right)\right)\right| \leq \frac{1}{2} \mathcal{X}_{1,0.95}^{2} \quad \text { whenever } p_{k} \in\left[p_{k}^{\min }, p_{k}^{\max }\right] .
$$

In (5.5), $\mathcal{L}\left(\mathbf{p}^{*}\right)$ stands for the likelihood function,

$$
\mathcal{L}(\tilde{\mathbf{p}}):=\max _{\mathbf{p} \in S\left(p_{k}\right)} \mathcal{L}(\mathbf{p}), \quad \text { where } S\left(p_{k}\right):=\left\{\left.\left[p_{1}, p_{2}, \ldots, p_{k-1}, p, p_{k+1}, \ldots, p_{n_{p}}\right]\right|_{p \text { fixed }}\right\},
$$

and $\mathcal{X}_{1,0.95}^{2}=3.841$ is the 0.95 th quantile of the $\mathcal{X}^{2}$-distribution for 1 degree of freedom.

The estimated $95 \%$ confidence intervals, cf. Table 3 and Fig. 7, were computed using a numerical algorithm presented in [18]. The profile-based CIs, except $\mathrm{CI}_{\alpha_{7}}$, are rather close to the variance-covariance-based ones. The fact that they turned out to be somewhat narrower for most of the parameters indicates that the objective function grows a bit faster than the parabolic one. 


\begin{tabular}{|c|c|c|c|c|c|c|c|c|c|c|}
\hline & $\alpha_{0}$ & $\alpha_{1}$ & $\alpha_{2}$ & $\alpha_{3}$ & $\alpha_{4}$ & $\alpha_{5}$ & $\alpha_{6}$ & $\alpha_{7}$ & $\beta_{4}$ & $\beta_{5}$ \\
\hline$\alpha_{1}$ & $-1.3 \mathrm{e}-1$ & & & & & & & & & \\
$\alpha_{2}$ & $1.8 \mathrm{e}-1$ & $-3.3 \mathrm{e}-1$ & & & & & & & & \\
$\alpha_{3}$ & $4.6 \mathrm{e}-1$ & $2.0 \mathrm{e}-1$ & $-1.6 \mathrm{e}-1$ & & & & & & & \\
$\alpha_{4}$ & $-1.6 \mathrm{e}-1$ & $-1.4 \mathrm{e}-1$ & $-3.5 \mathrm{e}-1$ & $-4.1 \mathrm{e}-1$ & & & & & \\
$\alpha_{5}$ & $9.0 \mathrm{e}-2$ & $8.0 \mathrm{e}-2$ & $6.0 \mathrm{e}-2$ & $1.0 \mathrm{e}-1$ & $-2.6 \mathrm{e}-1$ & & & & \\
$\alpha_{6}$ & $9.0 \mathrm{e}-2$ & $8.0 \mathrm{e}-2$ & $6.0 \mathrm{e}-2$ & $1.0 \mathrm{e}-1$ & $-2.2 \mathrm{e}-1$ & $9.8 \mathrm{e}-1$ & & & \\
$\alpha_{7}$ & $3.0 \mathrm{e}-2$ & $3.0 \mathrm{e}-2$ & $2.0 \mathrm{e}-2$ & $3.0 \mathrm{e}-2$ & $-8.0 \mathrm{e}-2$ & $3.6 \mathrm{e}-1$ & $3.7 \mathrm{e}-1$ & & & \\
$\beta_{4}$ & $4.6 \mathrm{e}-1$ & $3.1 \mathrm{e}-1$ & $3.3 \mathrm{e}-1$ & $5.5 \mathrm{e}-1$ & $-8.8 \mathrm{e}-1$ & $2.6 \mathrm{e}-1$ & $2.3 \mathrm{e}-1$ & $8.0 \mathrm{e}-2$ & & \\
$\beta_{5}$ & $-1.6 \mathrm{e}-1$ & $-1.2 \mathrm{e}-1$ & $-3.0 \mathrm{e}-1$ & $-3.5 \mathrm{e}-1$ & $9.2 \mathrm{e}-1$ & $-5.4 \mathrm{e}-1$ & $-5.1 \mathrm{e}-1$ & $-1.9 \mathrm{e}-1$ & $-8.5 \mathrm{e}-1$ & \\
$\delta$ & $1.8 \mathrm{e}-1$ & $1.0 \mathrm{e}-1$ & $-1.6 \mathrm{e}-1$ & $-4.0 \mathrm{e}-2$ & $6.9 \mathrm{e}-1$ & $-6.3 \mathrm{e}-1$ & $-6.0 \mathrm{e}-1$ & $-2.2 \mathrm{e}-1$ & $-4.5 \mathrm{e}-1$ & $8.3 \mathrm{e}-1$ \\
\hline
\end{tabular}

Table 4: Correlations between the best-fit parameter estimates of the heterogenous model (3.1) computed from the variance-covariance matrix.

The profile-likelihood-based method does not provide an estimate of the upper limit of the $\mathrm{CI}_{\alpha_{7}}$. Its value tends to infinity as the iterations of the computational algorithm continue. The reason is as follows. Using the relationship between the maximum likelihood and least-squares objective function, the expression (5.5) is equivalent to

$$
\left|\ln (\Phi(\tilde{\mathbf{p}}))-\ln \left(\Phi\left(\mathbf{p}^{*}\right)\right)\right| \leq \frac{1}{n_{d}} \mathcal{X}_{1,0.95}^{2} \quad \text { whenever } p_{k} \in\left[p_{k}^{\min }, p_{k}^{\max }\right]
$$

where

$$
\Phi(\tilde{\mathbf{p}}):=\min _{\mathbf{p} \in S\left(p_{k}\right)} \Phi(\mathbf{p}) .
$$

Then, using $\ln \left(\Phi\left(\mathbf{p}^{*}\right)\right) \approx 20.96$, the final expression for computing the $95 \% \mathrm{CI}_{p_{k}}$ is equivalent to

$$
\Phi(\tilde{\mathbf{p}}) \in\left[\Phi\left(\mathbf{p}^{*}\right), 1.41 \times 10^{9}\right] \quad \text { whenever } p_{k} \in\left[p_{k}^{\min }, p_{k}^{\max }\right] .
$$

Figure 8 depicts the profile of $\Phi(\tilde{\mathbf{p}})$ with respect to the parameters $\alpha_{0}$ and $\alpha_{7}$, varying within some ranges. For $\alpha_{0}$, the profile of $\Phi(\tilde{\mathbf{p}})$ shows a well balanced and symmetric behavior within the computed confidence interval $\left[\alpha_{0}^{\min }, \alpha_{0}^{\max }\right]$. We see that $\Phi(\tilde{\mathbf{p}})>$ $1.41 \times 10^{9}$ outside this interval. In contrast, the profile of $\Phi(\tilde{\mathbf{p}})$ is asymmetric for the parameter $\alpha_{7}$ with the right tail staying below $1.41 \times 10^{9}$ as $\alpha_{7} \rightarrow \infty$. The latter is in agreement with the behavior of the objective function $\Phi$ shown in Fig. 6 .

is also a term used in statistics to describe a variety of methods for computing summary statistics using subsets of available data (jackknife), drawing randomly with replacement from a set of data points (bootstrapping

\subsection{Bootstrap method}

The bootstrap technique is a computationally intensive method for estimating the mean and standard error on the basis of samples generated from small data sets (say of size $n$ ) [8]. 
The new data sets, being subsets of available data, are randomly drawn from an original observations set of data points and fit as if they were independent observations. Therefore, the method works by resampling randomly, for example with a uniform probability equal to $1 / n$, the observed sample values to model the unknown population of observations. The whole process of random samplings with replacement is repeated $M$ times to generate $M$ data sets. For these data sets, one computes the best-fit parameter estimates $\mathbf{p}_{m}^{*}, m=$ $1,2, \ldots, M$, which provide an estimate of the 'true' standard error by taking the standard deviation of the $M$ values of $\mathbf{p}_{m}^{*}$. The standard interval

$$
C I_{p_{k}}=\left[\mathcal{E}\left(p_{k}^{*}\right)-\sigma_{p_{k}} z(\theta), \mathcal{E}\left(p_{k}^{*}\right)+\sigma_{p_{k}} z(\theta)\right], \quad k=1,2, \ldots, n_{p},
$$

approximates the $100 \cdot \theta$ bootstrap confidence interval. Here $\mathcal{E}\left(p_{k}^{*}\right)$ and $\sigma_{p_{k}}$ are the mean and standard deviation, respectively, of the estimates of a parameter $p_{k}^{*}$ that are found by fitting the bootstrap resamples of the original data and $z(\theta)$ is the $100 \cdot \theta$-th percentile of a normal deviate. The value $z(0.95) \approx 1.96$ is used for approximating the $95 \% \mathrm{CI}$ in conjunction with a large number of resamplings $M$. We consider non-parametric and parametric approaches for samples generation.

\subsubsection{A non-parametric bootstrap}

We used a non-parametric bootstrap approach [7] as follows. The original observations are available for days 3 to 7 and characterize the numbers of live and dead cells. One can treat the measurements of live and dead lymphocytes at a given time as independent, whereas the distribution of the cells over the division number is a single entity which can not be split. Therefore, we considered five measurement times (days 3 to 7 ) for the live and dead cells as separate data points, which represent altogether the original sample set of size $2 n_{t}, n_{t}=5$. The resampled data sets were generated by choosing the measurement times randomly from the original set using a uniform probability distribution.

Let the $m$-th resampling procedure select the following set of measurement days $\left\{t_{0}^{N}, t_{1}^{N}, t_{2}^{N}, t_{3}^{N}, t_{4}^{N}\right\}$ for $N$ and $\left\{t_{0}^{D}, t_{1}^{D}, t_{2}^{D}, t_{3}^{D}, t_{4}^{D}\right\}$ for $D$. The day set is further ordered to ensure that $t_{0}^{N} \leq t_{1}^{N} \leq t_{2}^{N} \leq t_{3}^{N} \leq t_{4}^{N}$ and $t_{0}^{D} \leq t_{1}^{D} \leq t_{2}^{D} \leq t_{3}^{D} \leq t_{4}^{D}$. The resamples consisting of only one day of measurement, i.e., the measurement day represented $n_{t}$ times, are discarded from analysis as being not informative. The bootstraping analysis involves the following heuristics:

- construct samples of measurements by selecting the measurement times and corresponding cell data at the sampled days;

- take the best-fit parameter vector as an initial guess for fitting the resampled data;

- compute the best-fit estimate $\tilde{\mathbf{p}}^{*}$ using the objective function

$$
\tilde{\Phi}(\mathbf{p})=\sum_{i=1}^{4}\left(\sum_{j=0}^{7}\left(N_{j}^{t_{i}^{N}}-N_{j}\left(t_{i}^{N} ; \mathbf{p}\right)\right)^{2}+\left(D^{t_{i}^{D}}-D\left(t_{i}^{D} ; \mathbf{p}\right)\right)^{2}\right),
$$


where $N_{j}^{t_{i}^{N}}$ and $D^{t_{i}^{D}}$ are the original data at the resampled times $t_{i}^{N}$ and $t_{i}^{D}$, respectively. If it happens that $t_{0}^{D}<t_{0}^{N}$, we compute the model predictions for $N_{j}$ for all times $t_{i}^{D}$ for which $t_{i}^{D}<t_{0}^{N}, i=0, \ldots$.

Repeating the above bootstrapping procedure $M$ times, we compute a set of best-fit vectors $\tilde{\mathbf{p}}_{m}^{*}, m=1, \ldots, M$, for $M$ bootstrap samples. The standard deviation of the element $p_{k}$ of the vector $\tilde{\mathbf{p}}_{m}^{*}$ can be approximated from the above set in a usual way,

$$
\sigma_{p_{k, M}}=\left(\frac{\sum_{m=1}^{M}\left|\tilde{p}_{k, m}^{*}-\hat{p}_{k}^{*}\right|^{2}}{M-1}\right)^{1 / 2}, \quad \text { where } \quad \hat{p}_{k}^{*}:=\frac{\sum_{m=1}^{M} \tilde{p}_{k, m}^{*}}{M},
$$

cf. [7], equation (2.4). If

$$
\lim _{M \rightarrow \infty} \sigma_{p_{k, M}}=: \sigma_{p_{k}}
$$

exists, then $\sigma_{p_{k}}$ is the bootstrap estimate of the standard deviation for $p_{k}$. In this case, a standard bootstrap $95 \%$ CI for $p_{k}$ is given by

$$
C I_{p_{k, M}}=\left[\hat{p}_{k}^{*}-1.96 \sigma_{p_{k, M}}, \hat{p}_{k}^{*}+1.96 \sigma_{p_{k, M}}\right] .
$$

It is important that $M$ is large enough to achieve convergence of $\sigma_{p_{k, m}}$ as $m \rightarrow M$. However, as it was noticed in [7], the bootstrap is not generally reliable for small sample sizes regardless of how many resamples $M$ are used.

The computed $95 \%$ CIs for the parameters of the heterogenous model are presented in Table 3 and Fig. 7. These results are based on $M=4000$ resamples. The bootstrap CIs for all parameters appear to be much broader than the ones predicted with variance-covariance and profile-likelihood-based methods. As shown in Fig. 9, the standard deviation estimates keep fluctuating, although to a different extent depending on particular parameter. A slow convergence of the estimates can be seen for all the model parameters, except $\alpha_{6}$ and $\alpha_{7}$, and the evolution of $\sigma_{\alpha_{0}, M}$ and $\sigma_{\beta_{4}, M}$ provides two representative examples. Due to the small size of the original data, the use of the large number of resamples does not ensure a faster convergence and the sample estimates of $\sigma$ were obtained with only 1 or 2 significant digits. The observed jumps in the evolution of $\sigma_{\alpha_{6}, M}$ and $\sigma_{\alpha_{7}, M}$ could be due to the specific features of CFSE data set: $(i)$ the contribution of the data on cells which have undergone 6 and 7 divisions to the value of the objective function is much smaller compared to the other cellular compartments; ( $i i)$ the parameters $\alpha_{6}$ and $\alpha_{7}$ influence fewer solution components than other parameters and are, therefore, less well defined; ( $i i i)$ the non-parametric bootstrap approach allows very "sparse" data sets, e.g. days $\{3,3,3,3,5\}$, which carry rather little information about the cell kinetics.

\subsubsection{A parametric bootstrap}

Applying a parametric variant of the bootstrap method to estimate the 95\% CIs for the best-fit values of the heterogenous model parameters, new data samples were generated by perturbing the original data as follows,

$$
\begin{aligned}
\tilde{N}_{j}^{i} & =N_{j}^{i}+\sigma^{*} \mathcal{N}(0,1), \\
\tilde{D}^{i} & =D^{i}+\sigma^{*} \mathcal{N}(0,1), \quad i=0,1, \ldots, 4, \quad j=0,1, \ldots, 7,
\end{aligned}
$$


where $N_{j}^{i}$ and $D^{i}$ are the data from Table 1 at time $t_{i}, \mathcal{N}(0,1)$ is a normally distributed random variable with zero mean and variance equal to one, and $\sigma^{*} \approx 5.9 \times 10^{3}$, cf. (4.3).

The maximum likelihood estimate of the variance $\sigma^{*^{2}}$ is rather large compared to the values of $N_{7}^{i}$. Hence, the parametric resampling (5.12) can generate biologically improper values of $\tilde{N}_{7}^{i}$, e.g. negative, sharply decreasing values. Such inconsistent data for $\tilde{N}_{7}^{i}$ were filtered out by ignoring the sequences that do not increase monotonically. The above difficulty indicates that the variance in the components of the observed state space vector might not be equal. Rather, $\sigma^{*^{2}}$ should be dependent on the number of divisions cells have undergone and therefore, estimated separately.

For each perturbed data sample, we computed the bootstrap estimates of the best-fit parameters by minimizing the following objective function

$$
\tilde{\Phi}(\mathbf{p})=\sum_{i=1}^{4}\left(\sum_{j=0}^{7}\left(\tilde{N}_{j}^{i}-N_{j}\left(t_{i} ; \mathbf{p}\right)\right)^{2}+\left(\tilde{D}^{i}-D\left(t_{i} ; \mathbf{p}\right)\right)^{2}\right) .
$$

Similar to the non-parametric bootstrap, we generated 4000 data samples. The bootstrap set of the best-fit parameter values was used to calculate, by (5.10) and (5.11), the standard deviation of the estimators to approximate the $95 \%$ CIs, cf. Table 3 and Fig. 7. The estimated CIs are consistent with those given by the variance-covariance and profilelikelihood methods. The convergence of all computed standard deviations is similar to the ones shown in Fig. 9 (left), $\sigma_{\alpha_{6}, M}$ and $\sigma_{\alpha_{7}, M}$ evolve without jumps and converge to smaller values than the ones estimated by the first two methods. We believe that this is due to the filtering of the generated data $\tilde{N}_{7}$ as we discussed above.

\subsection{Computational performance}

The data fitting and confidence interval analysis of the parameters are computationally intensive procedures. All computations were carried out using MATLAB 7.0 routines on PC Pentium $4 \mathrm{CPU} 2.26 \mathrm{GHz}$. It is instructive to analyze the relative performance of the computational approaches to CIs analysis that we used. To this end we look at the estimates of the CPU time required to perform the CIs analysis of the 11 estimated parameters of the heterogenous model (3.1) by the three methods. The CPU time was measured by the Matlab code cputime.

To solve the minimization problem, we used the Matlab code fminsearch based on the Nelder-Mead simplex (direct search) method. The CPU time needed to find a solution of one minimization problem for model $(3.1), t_{\min }$, is given in Table 5 for different values of the required accuracy defined by the fminsearch parameters TolFun and TolX. This time practically does not depend on the requested accuracy since analytical solutions to the model were used. However, it depends on how close the initial guess is to a local or global minimum. Note that the lower bound of the given time intervals corresponds to the initial parameter values close to the best-fit estimate (the first two significant digits of the best-fit parameter values remain unchanged), and the upper bound corresponds to a poor initial guess. 


\begin{tabular}{|c|c|c|c|}
\hline Tolerances & $\begin{array}{c}\text { TolFun }=10^{-6} \\
\text { TolX }=10^{-6}\end{array}$ & $\begin{array}{c}\text { TolFun }=10^{-9} \\
\text { TolX }=10^{-9}\end{array}$ & $\begin{array}{c}\text { TolFun }=10^{-12} \\
\text { TolX }=10^{-12}\end{array}$ \\
\hline CPU time & $23-104$ & $26-108$ & $29-111$ \\
\hline
\end{tabular}

Table 5: The computational time $t_{\min }$ (seconds) required for solving one minimization problem by the Nelder-Mead simplex method.

The variance-covariance matrix based technique. The CPU time required to calculate CIs by the variance-covariance matrix based approach is determined by the time $t_{H}$ needed to compute the Hessian matrix (5.3). For our problem, $t_{H}$ is about 6 seconds and it does not depend on the requested accuracy since the matrix elements are computed using analytical first- and second-order partial derivatives of the solution with respect to the model parameters.

The profile-likelihood-based method. To compute the confidence intervals by the profile-likelihood-based method, we used an algorithm described in [18], which is based on a modified Newton-Raphson iteration to solve a system of equations that determines the lower and upper bounds of a CI. One bound is usually located by 4-10 iterations, depending on the requested accuracy and the quality of the starting point. The computational cost of a single iteration is determined by the time needed to evaluate the first- and secondorder partial derivatives of the likelihood function with respect to the parameters being optimized, i.e. by the time $t_{H}$. Hence, the CPU time to compute CIs for 11 parameters can be estimated as

$$
t_{H} \times\left(\sum_{i=1}^{11} \sum_{j=1}^{2} n_{i, j}\right), \quad 4 \leq n_{i, j} \leq 10,
$$

where $n_{i, 1}$ and $n_{i, 2}$ specify the number of iterations needed to compute the low and upper bounds of the CI for the $i$-th parameter, respectively.

The bootstrap approach. The computational cost of the bootstrap method (both parametric and non-parametric) is determined by the following factors: $(i)$ the total number $M$ of resamples ( $M=4000$ in our case), (ii) the number of minimization runs, $s_{m}, m=1, \ldots, M$, which are needed to compute a satisfactory best-fit minimum (hopefully, a global one) for every generated data set, and (iii) the time required for solving one minimization problem, $t_{\text {min }}$. Hence, the total CPU time can be evaluated by

$$
t_{\text {min }} \times \sum_{m=1}^{M} s_{m}, \quad s_{m} \geq 1 .
$$

Clearly, the bootstrap analysis becomes computationally highly expensive for a large number of resamplings $M$ and/or when $s_{m} \gg 1$. The latter takes place when, e.g., a model parameter is poorly defined by the data used. 
Summary. Table 6 summarizes the CPU time needed to compute the confidence intervals for the estimated parameters by the three methods we used. The methods can be ranked in terms of their computational demands as follows: variance-covariance technique is superior to the profile-likelihood method which is (much) faster than the bootstrap. The time needed for the bootstrap approach is about 25 to 123 hours, even in a favorable case when the minimization problem is solved only once for each data sample, i.e., $s_{m}=1, m=1, \ldots, M$.

\begin{tabular}{|l|c|c|c|}
\hline Method & $\begin{array}{c}\text { Variance-covariance } \\
\text { matrix approach }\end{array}$ & $\begin{array}{c}\text { Profile-likelihood } \\
\text { method }\end{array}$ & $\begin{array}{c}\text { Bootstrap } \\
\text { method }\end{array}$ \\
\hline CPU & $t_{H}$ & $t_{H} \times\left(\sum_{i=1}^{11} \sum_{j=1}^{2} n_{i, j}\right)$ & $t_{\min } \times \sum_{m=1}^{M} s_{m}$ \\
time & 6 & $528-1320$ & $\begin{array}{c}92000-444000 \\
\text { case } s_{m}=1, m=1, \ldots, M\end{array}$ \\
\hline
\end{tabular}

Table 6: The total computational time (seconds) required for evaluating $95 \%$ confidence intervals for the estimated parameters of the heterogenous model (3.1) by the three methods.

\section{Kendall model vs Smith-Martin model on in vitro and in vivo data}

The heterogenous compartmental model (3.1) for cell kinetics was applied to CFSE data analysis generated by in vitro assays. To understand limitations of this model in a broader context, we compare its performance with a version of the Smith-Martin (SM) model of cell kinetics by considering both our in vitro data (Table 1) and the in vivo data presented in $[9]$.

\subsection{A version of Smith-Martin model}

The Smith-Martin model [16] is a well-known biological model for cell data analysis, in which the cell cycle stages are lumped into two states: the first state $(A)$ corresponds to the $G_{1}$ phase of the cell cycle and the second state $(B)$ represents the $S-G_{2}-M$ phases of the cycle. The progression through the cell cycle is assumed to have stochastic and deterministic components: the recruitment of cells from the $A$ state into $B$, respectively, a fixed-time progression through the $B$ state. In recent studies [6,9], a delay-type version of this model was proposed, which describes the rate of change of the populations of $\mathrm{T}$ lymphocytes in the $A$ and $B$ states, $A_{j}(t)$, respectively $B_{j}(t)$, that have undergone $j$ divisions,

$$
\begin{aligned}
& \frac{d A_{j}}{d t}(t)=2 \lambda A_{j-1}(t-\Delta) \mathrm{e}^{-d_{B} \Delta}-\left(\lambda+d_{A}\right) A_{j}(t), \quad j=1, \ldots, \infty, \\
& B_{j}(t)=\lambda \int_{0}^{\Delta} A_{j}(t-s) \mathrm{e}^{-d_{B} s} d s, \quad j=1, \ldots, \infty .
\end{aligned}
$$


The parameters of the model characterize the division $(\lambda)$ and death $\left(d_{A}, d_{B}\right)$ rates and a time lag $(\Delta)$ of transit through the $B$ state, see $[6,9]$ for details. In [9], the model variables $A_{j}(t)+B_{j}(t)$ were fitted to in vivo data on proliferation of $\mathrm{P}-14 \mathrm{Tg}$ naive CD8 $\mathrm{T}$ cells after adoptive transfer into an irradiated host, taken from [13] and summarized in Table 3 in [9]. A partly heterogenous version of the above model, in which the division and death rates of naive cells are different from those of the divided cells, was studied in [6] on in vitro data from [10].

\subsection{In vitro cell growth}

We used the Smith-Martin model (6.1) to assimilate the cell kinetics data presented in Table 1. The fitting procedure was the same as for the heterogenous compartmental model.

The best-fit solutions of the both models together with the experimental data for the total number of lymphocytes over all divisions, the mean value and the variance of the number of divisions lymphocytes have undergone are presented in Fig. 10. The cell growth pattern, which decelerates with time, indicates that the system is not in the phase of the 'steady state of exponential growth'. Whereas the heterogenous model is consistent with the data (the concave behavior), the solution of the SM model deviates quite substantially displaying a convex pattern of increase in the total cell number. Analogous can be seen for the predicted evolution of the mean value of cell population divisions for the SM model: it is a linear rather than a logistic-type behavior. The kinetics of the variance in the mean division number is a problem for both models, although the heterogenous model gives a much closer fit to the data.

The best-fit parameter estimates of the homogenous SM model are as follows: $A_{0}(0) \approx$ $6.73 \times 10^{4}, \lambda \approx 3.62 \times 10^{-2}, d_{A} \approx 8.28 \times 10^{-3}, d_{B} \approx 0, \Delta \approx 13.77$. The objective function value is $\Phi\left(\mathbf{p}^{*}\right) \approx 3.82 \times 10^{10}$. These estimates imply that there is little death in both states of the cell cycle. The numbers suggest that the average division time of cells (we follow the notation in [9]) is $T=1 / \lambda+\Delta \approx 41.4$ (hours), whereas the growth rate is $1 / T \approx 2.42 \times 10^{-2}$. These values are somewhat different from those predicted by the heterogenous and homogenous versions of the Kendall's model. The predicted fraction of responding cells $33.6 \%$ is close to our estimate $38 \%$. To characterize the information loss of the SM model for the given data set, we estimated the Akaike index. Its value being about 989 is higher than that of the heterogenous (732) and homogenous (799) versions of the Kendall model. Notice that the data $D(t)$ were not used in the parameter estimation for the last two models.

Overall, the above implies that the Kendall model performs better on the in vitro data which represent saturating growth of cell populations.

\subsection{In vivo cell growth}

The cell growth kinetics in vivo is characterized by data from [13] summarized in Table 3 in [9]. This data set was used to estimate the parameters of the heterogenous compartmental model. The best-fit results are summarized in Fig. 11. As a reference solution, we also present the fitting results obtained with the Smith-Martin model (6.1), for which 
the minimized objective function takes a slightly bigger value, $\Phi\left(\mathbf{p}^{*}\right) \approx 3.90 \times 10^{9}$ versus $\Phi\left(\mathbf{p}^{*}\right) \approx 2.38 \times 10^{9}$ for the heterogeneous model. The heterogenous model is consistent with the data on the total numbers of life lymphocytes and responding precursors, cf. Fig. 11a. The evolution of the mean number of cell divisions and its variance deviates from the observed numbers, especially at the earlier phase of cell proliferation, cf. Fig. 11b. According to the heterogenous model, the division rate is not uniform but depends on the proliferation history, cf. Fig. 11c. The death rate is close to zero, except the naive cell compartment, cf. Fig. 11d. The proliferation rate estimate by the SM model, $1 / T$, is close to that of the heterogenous model for naive cells and the cells which have undergone 1 and 2 divisions but deviates essentially for cells generated later, cf. Fig 11c.

The CIs for the optimized parameters were estimated by the variance-covariance matrix method and the prolife-likelihood approach. Whereas the ranges estimated using the two methods appear to be close to each other for the Smith-Martin model, the corresponding CI estimates differ for the heterogenous model. The fact that intervals predicted by the variance-covariance technique are larger than those by the profile-likelihood method, suggests that the objective function grows faster than its parabolic approximation. The Akaike measures of the information loss are $\mu_{c A I C} \approx 551$ and $\mu_{c A I C} \approx 631$ for the heterogenous and SM models, respectively.

\subsection{Robustness to variations in data set}

We examined the robustness of the parameter estimates with respect to the addition or deletion of observations at the last measurement time. For a heterogenous model, the effect of the perturbation is localized towards the older generation cell compartments. For example, the deletion of day 7 data (last raw in Table 1) from the least-squares function fitting led to the following changes in the best-fit values: five out of 11 identified parameters varied within $1-10 \%$, i.e. the parameters $\left(\alpha_{j}, j=1, \ldots, 4\right.$, and $\left.\delta\right)$, and the rests varied from $14 \%$ to $47 \%$.

For comparison, we did a similar analysis of the version of the Smith-Martin model from [6] that considers the division and death rates of cells which have undergone at least one division as being independent of further divisions. In this work, a set of in vitro data on CD4 T cell proliferation stimulated with anti-CD3 antibodies [10], covering the range from 60 through 96 hours, was analyzed. If one extends the data set used for parameter estimation in [6] by adding the data at $t=96$ hours (this measurement was not used for the parameter estimation of the Smith-Martin model in [6]), then the change in the best-fit parameter values is more substantial, ranging from $4 \%$ to $550 \%$.

\section{Conclusions}

We have presented a comprehensive computational analysis of the human lymphocyte kinetics from CFSE data. The data set we used is typical for CFSE labelling assay and includes the total number of live and dead (but not disintegrated) cells. We applied a heterogenous compartmental model, first proposed by Kendall for cell cycle progression modelling, to estimate, following the maximum likelihood approach, the proliferation and 
death rates of PHA-stimulated T cells in vitro. The model predicts that the turnover rates are division number dependent. Similar analysis of in vivo data on the dynamics of P-14 tg CTL after adoptive transfer into irradiated mice [13] confirmed our findings. Therefore, the use of homogenous models for the analysis of cell kinetics deserves a caution.

Our experience indicates that a reliable estimation of the heterogenous model parameters is limited to those cell compartments which have undergone the number of division cycles which is two divisions less then the maximum number of divisions followed in the CFSE assay. For example, the parameter, characterizing the division rate of cells which have undergone the maximal number of divisions $\left(n_{\max }\right)$, is exceptional in the sense that it can not be identified reliably from finite series of data covering the division range from 0 to $n_{\max }$.

For the first time, three major methods of the analysis of the confidence in the best-fit parameter estimates: the variance-covariance matrix based approach, the profile-likelihood based method and the bootstrap (non-parametric and parametric) method, were compared in terms of their consistency and computational performance. The presented results show that the three techniques give rather close estimates of the $95 \%$ confidence intervals of the identified parameters of the considered heterogenous model.

The non-parametric bootstrap approach gave larger confidence intervals compared to the other methods. We explain this discrepancy by the sparsity of the original data, provided that measurements are made at few time points, many resampled data are characterized by repetition of the same data. The least computationally demanding method of the CIs analysis is the variance-covariance method. However, the underlying parabolic approximation of the objective function might not always be an appropriate one. The profile-likelihood-based approach seems to be the technique which provides a reasonable balance between the accuracy and computational cost.

We showed that the information-theoretic criteria, which take into account the accuracy (bias) of the data fitting and the complexity of the mathematical model, provide a rigorous basis for ranking various models. We computed the corrected Akaike index for the models considered in the present study: the heterogenous and homogenous compartmental models as variants of the Kendall model and a variant of the homogenous Smith-Martin model, to assess their distance from the 'full truth' model of the given data. The heterogenous Kendall model gave the least information loss for the type of data we considered.

The computational methodology implemented in this study can be further tailored to the needs of practical research on cell kinetics which uses CFSE labelling. Together with appropriate mathematical models, it should lead to a better understanding of the parameters of lymphocyte responsiveness under normal and pathological conditions.

\section{Acknowledgements}

This work was partially supported by the Leverhulme Trust, the Russian Foundation of Basic Research (RFBR-05-01-00732) and the Programme "Bilateral scientific cooperation with Flanders" (RFBR-MF 05-01-02853-MF-a). 


\section{References}

[1] N. R. Abu-Absi, A. Zamamiri, J. Kacmar, S. J. Balogh, and F. Srienc. Automated flow cytometry for acquisition of time-dependent population data. Cytometry A, 51:87-96, 2003.

[2] C. T. H. Baker, G. A. Bocharov, J. M. Ford, P. M. Lumb, S. J. Norton, C. A. H. Paul, T. Junt, and B. Ludewig P. Krebs. Computational approaches to parameter estimation and model selection in immunology. J. Comput. Appl. Math., 184:50-76, 2005 .

[3] C. T. H. Baker, G. A. Bocharov, C. A. H. Paul, and F. A. Rihan. Computational modelling with functional differential equations: identification, selection and sensitivity. Appl. Numer. Math., 53:107-129, 2005.

[4] Y. Bard. Nonlinear parameter estimation. Academic Press, New York, 1974.

[5] K. P. Burnham and D. R. Anderson. Model selection and multimodel inference - a practical information-theoretic approach, 2nd ed. Springer-Verlag, New York, 2002.

[6] R. J. De Boer and A. S. Perelson. Estimating division and death rates from CFSE data. J. Comput. Appl. Math., 184(1):140-164, 2005.

[7] B. Efron and R. Tibshirani. Bootstrap methods for standard errors, confidence intervals, and other measures of statistical accuracy. Stat. Sci., 1(1):54-77, 1986.

[8] B. Efron and R. Tibshirani. Introduction to the bootstrap. Chapman and Hall, New York, 1993.

[9] V. V. Ganusov, S. S. Pilyugin, R. J. De Boer, K. Murali-Krishna, R. Ahmed, and R. Antia. Quantifying cell turnover using CFSE data. J. Immunol. Methods, 298:183$200,2005$.

[10] A. V. Gett and P. D. Hodgkin. A cellular calculus for signal integration by T cells. Nat. Immunol., 1:239-244, 2000.

[11] H. Gudmundsdottir, A. D. Wells, and L. A. Turka. Dynamics and requirements of $\mathrm{T}$ cell clonal expansion in vivo at the single-cell level: effector function is linked to proliferative capacity. J. Immunol., 162:5212-5223, 1999.

[12] D. G. Kendall. On the role of variable generation time in the development of a stochastic birth process. Biometrika, 35:316-330, 1948.

[13] K. Murali-Krishna and R. Ahmed. Cutting edge: naive T cells masquerading as memory cells. J. Immunol., 165:1733-1737, 2000.

[14] S. S. Pilyugin, V. V. Ganusov, K. Murali-Krishna, R. Ahmed, and R. Antia. The rescaling method for quantifying the turnover of cell populations. J. theor. Biology, $225(2): 275-283,2003$. 
[15] S. I. Rubinov. Cell kinetics. In L. A. Segel, editor, Mathematical models in molecular and cellular biology, pages 502-522. Cambridge University Press, Cambridge, 1980.

[16] J. A. Smith and L. Martin. Do cell cycle? Proc Nat. Acad. Sci. USA, 70(4):1263-1267, 1973.

[17] H. Veiga-Fernandes, U. Walter, C. Bourgeois, A. McLean, and B. Rocha. Response of naive and memory $\mathrm{CD}^{+} \mathrm{T}$ cells to antigen stimulation in vivo. Nat. Immunol., 1:47-53, 2000 .

[18] D. J. Venzon and S. H. Moolgavkar. A method for computing profile-likelihood-based confidence intervals. Appl. Statist., 37(1):87-94, 1988.

[19] A. D. Wells, H. Gudmundsdottir, and L. A. Turka. Following the fate of individual $\mathrm{T}$ cells throughout activation and clonal expansion. Signals from T cell receptor and CD28 differentially regulate the induction and duration of a proliferative response. $J$. Clin. Invest., 100:3173-3183, 1997. 


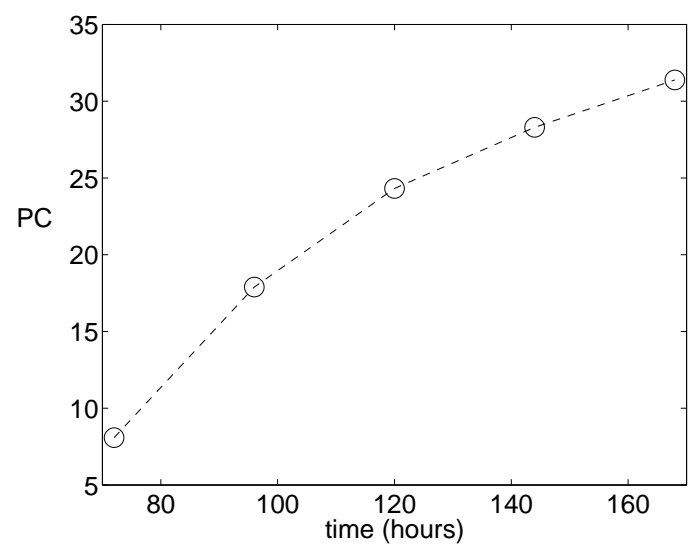

Figure 1: Proliferative capacity (o) of the human peripheral blood mononuclear cell population following stimulation with PHA in vitro. It characterizes the average number of daughter cells generated per responding precursor by time $t$. Estimated from Table 1 data under the assumption of no cell death.
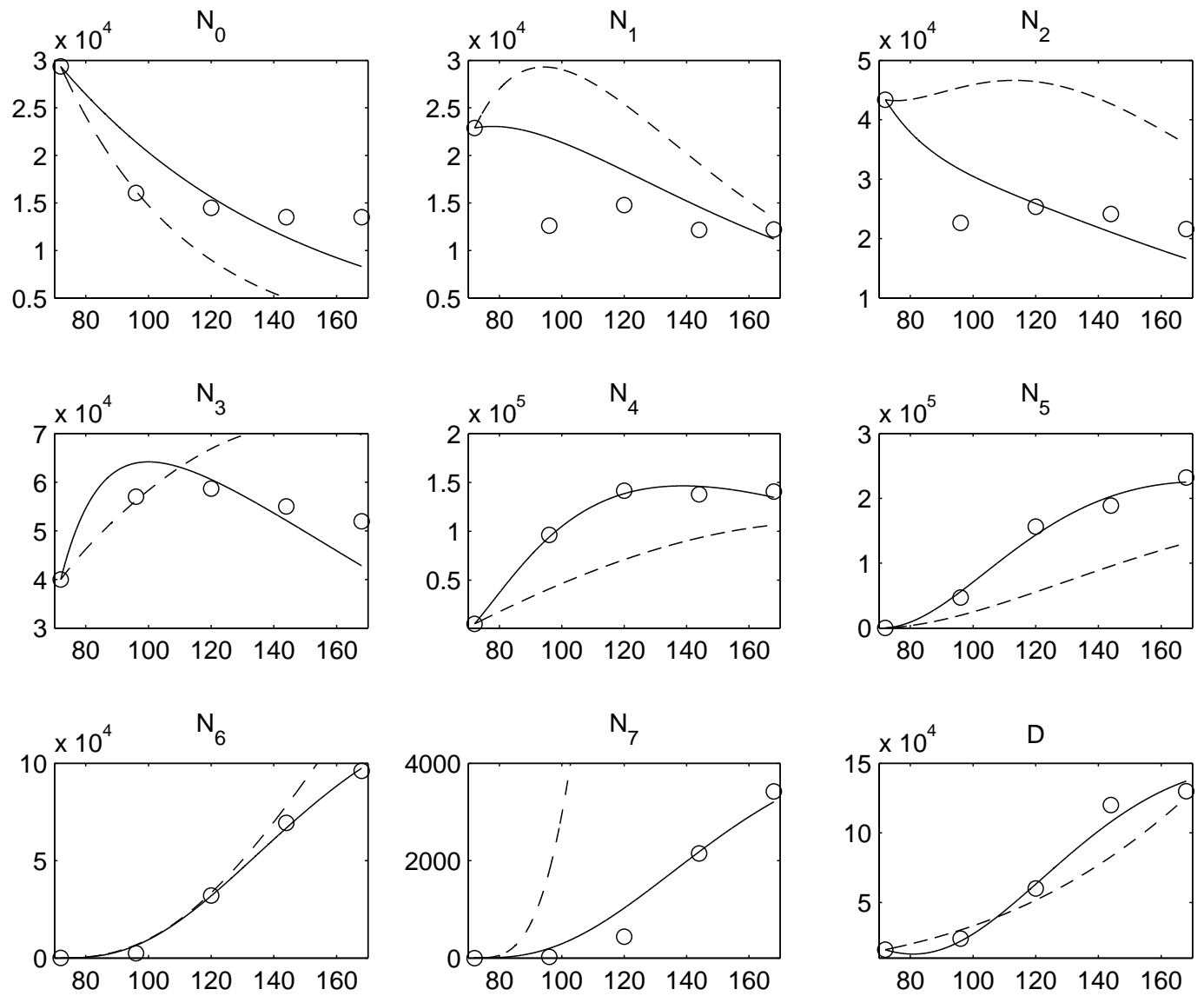

Figure 2: Experimental data (o) and the best-fit solutions of the heterogenous (solid lines) model (3.1) and the homogenous (dashed lines) model (3.3). 

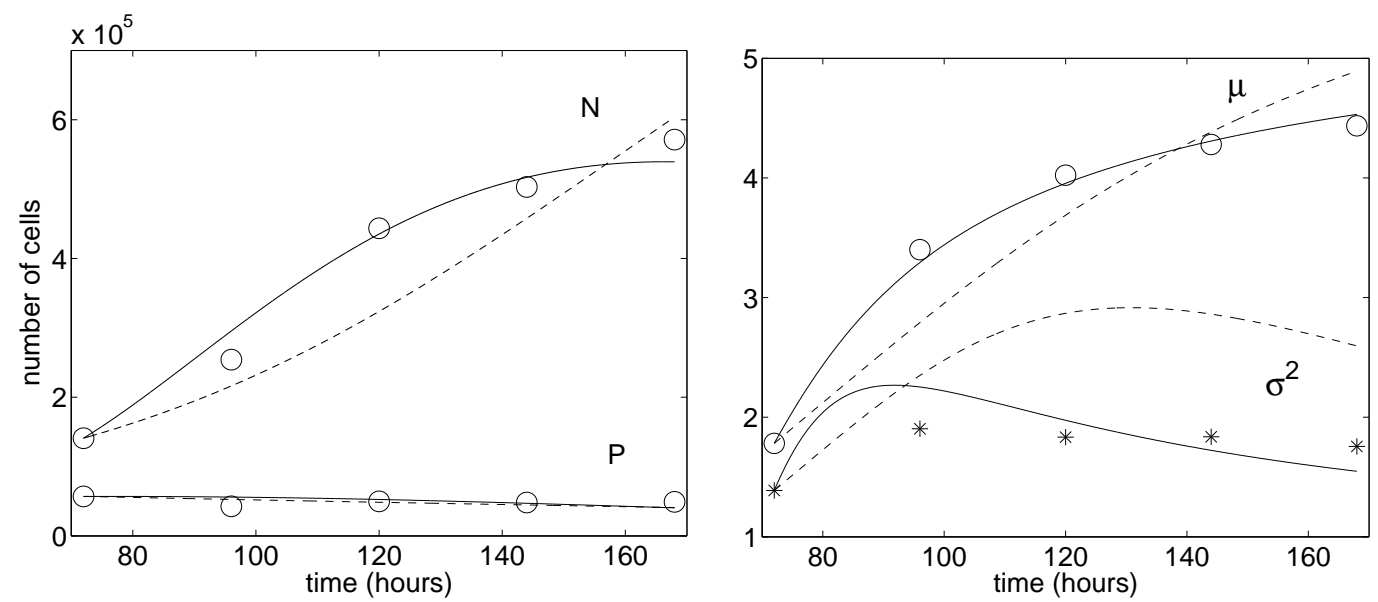

Figure 3: The kinetics of the PBMC population growth. Left: The total number of live lymphocytes $N$ over all divisions and the number of precursors $P$, that would have generated the current lymphocyte population in the absence of death, estimated from the data in Table 1 ( $\circ$ ) and predicted by the heterogenous (solid line) model (3.1) and the homogenous (dashed line) model (3.3) for the best-fit parameter values. Right: The behavior of the mean division number $\mu(0)$ and its variance $\sigma^{2}(*)$ suggested by the data. The solid and dashed curves correspond to $\mu(t)$ and $\sigma^{2}(t)$ computed using the best-fit solutions of the heterogeneous and homogeneous models, respectively.
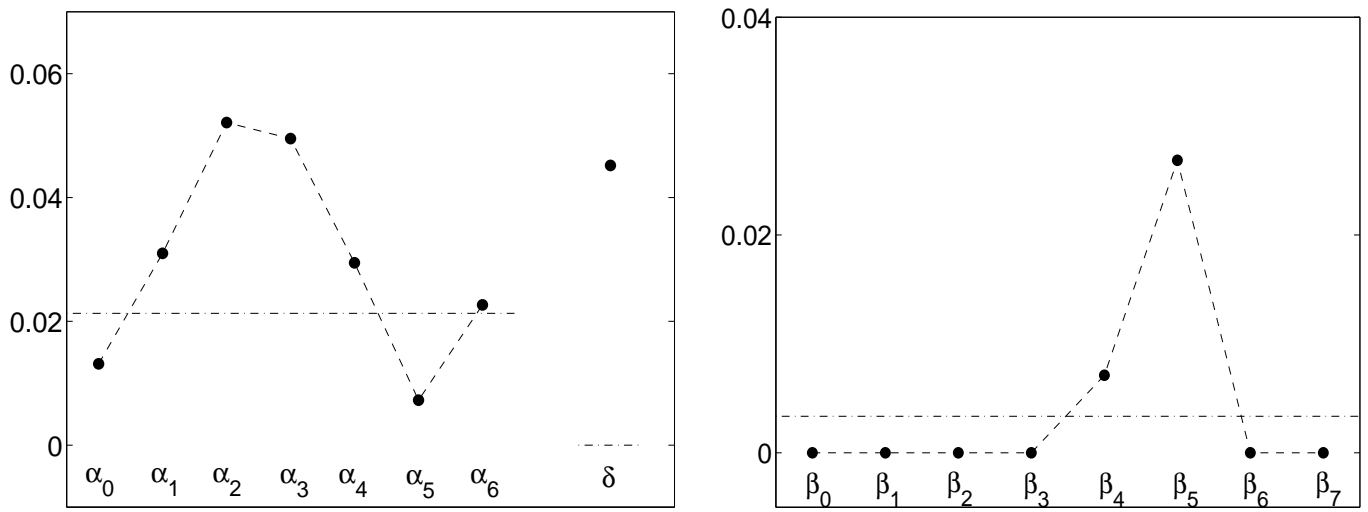

Figure 4: Dependence of the cell turnover parameters on the division number. The best-fit estimates of the division rate (left) and the death rate (right) parameters of the heterogeneous $(\bullet)$ and homogeneous (dot-dashed curves) models. The best-fit value of $\alpha_{7}, \alpha_{7}^{*} \approx 1.37$, is not shown. 

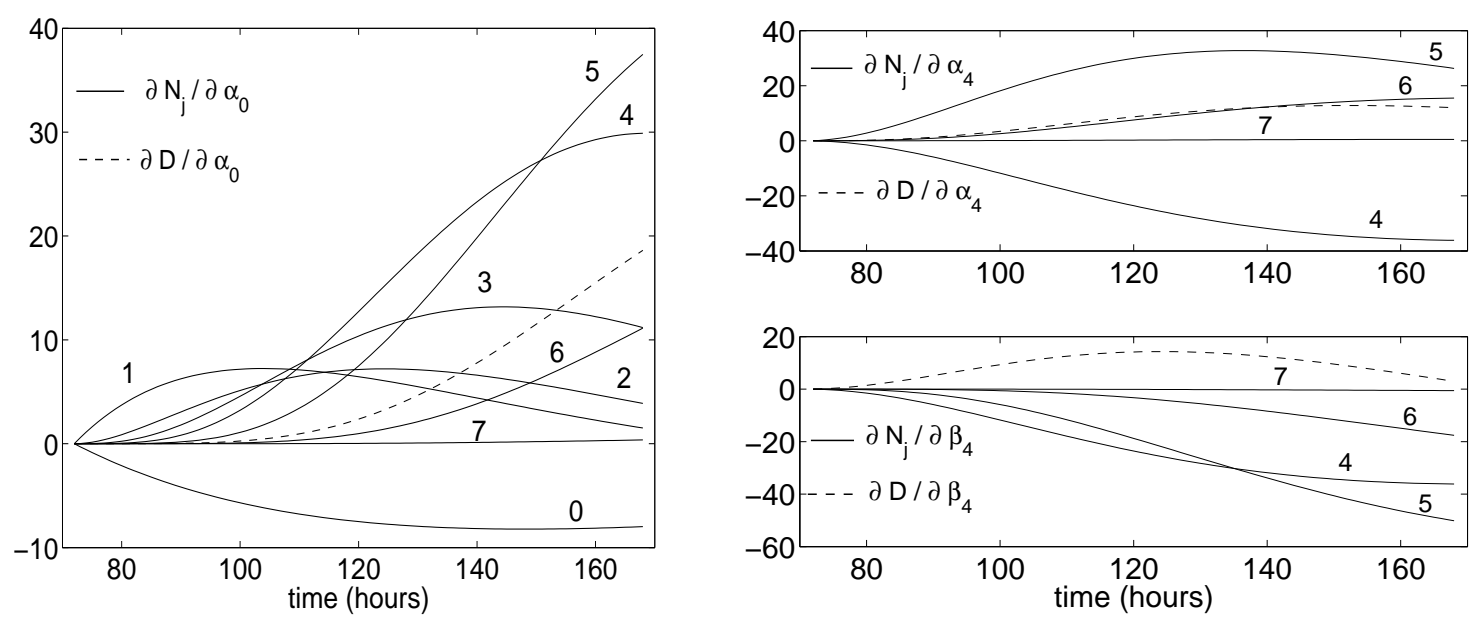

Figure 5: Sensitivity coefficients computed for the best-fit solution of the heterogenous model with respect to the division rate of naive cells (left) and the division and death rates of cells which have undergone 4 divisions (right). The numbers near the curves correspond to the index $j$ of $N_{j}$. 

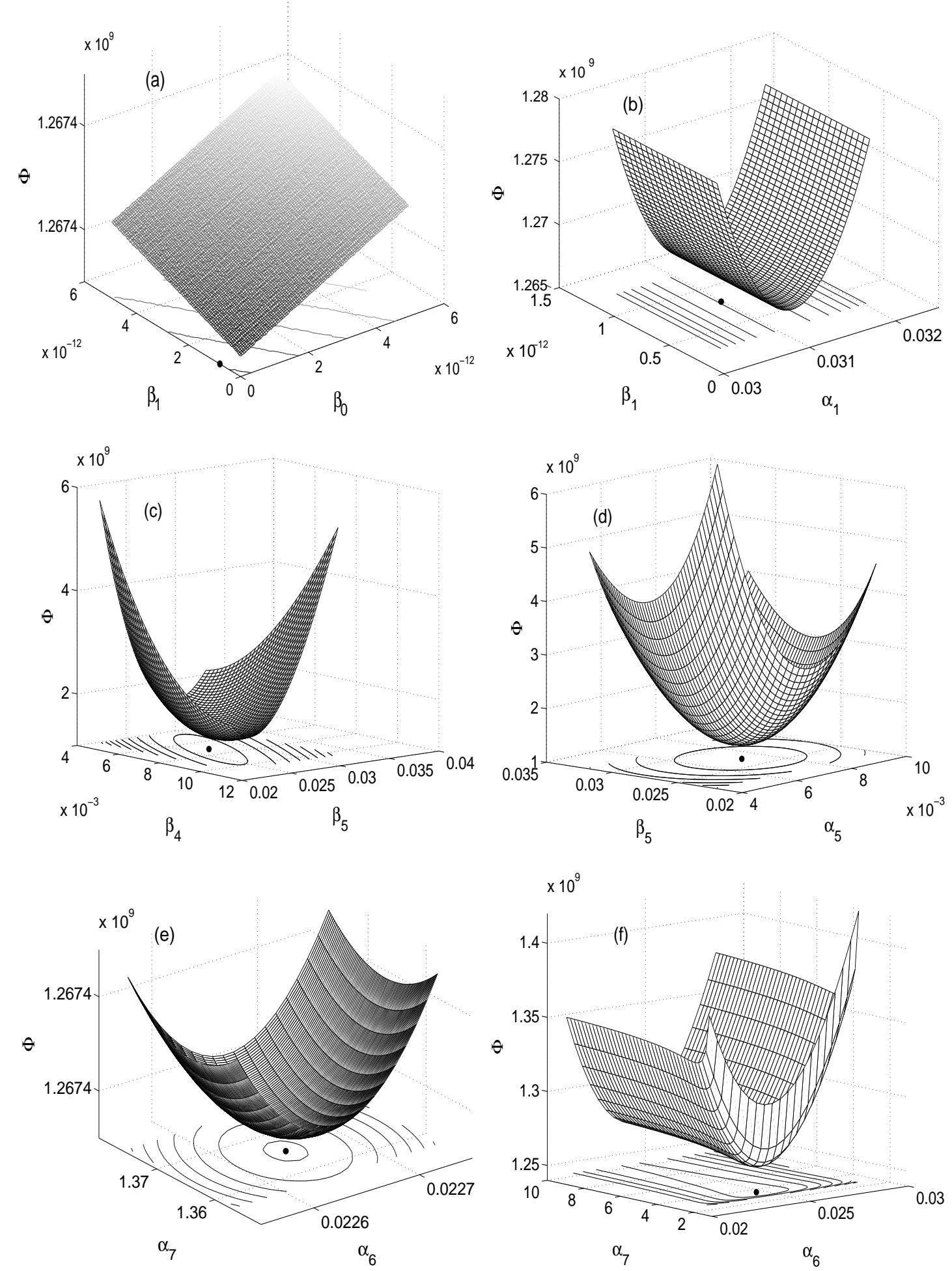

Figure 6: The behavior of the least-squares objective function $\Phi$ (with contour plots) in the neighborhood of the computed minimum $(\bullet)$ for various pairs of the model parameters. The other parameters are kept fixed at their best-fit values. A parabolic shape (figures (c)-(d)) indicates a locally well-defined minimum. The plane-type behavior (a) suggests that the extremum is attained at the border of the feasible region (non-negatize parameter values domain), whereas valley-type contours (figures (b) and (f)) indicate a poorly defined minimum. 

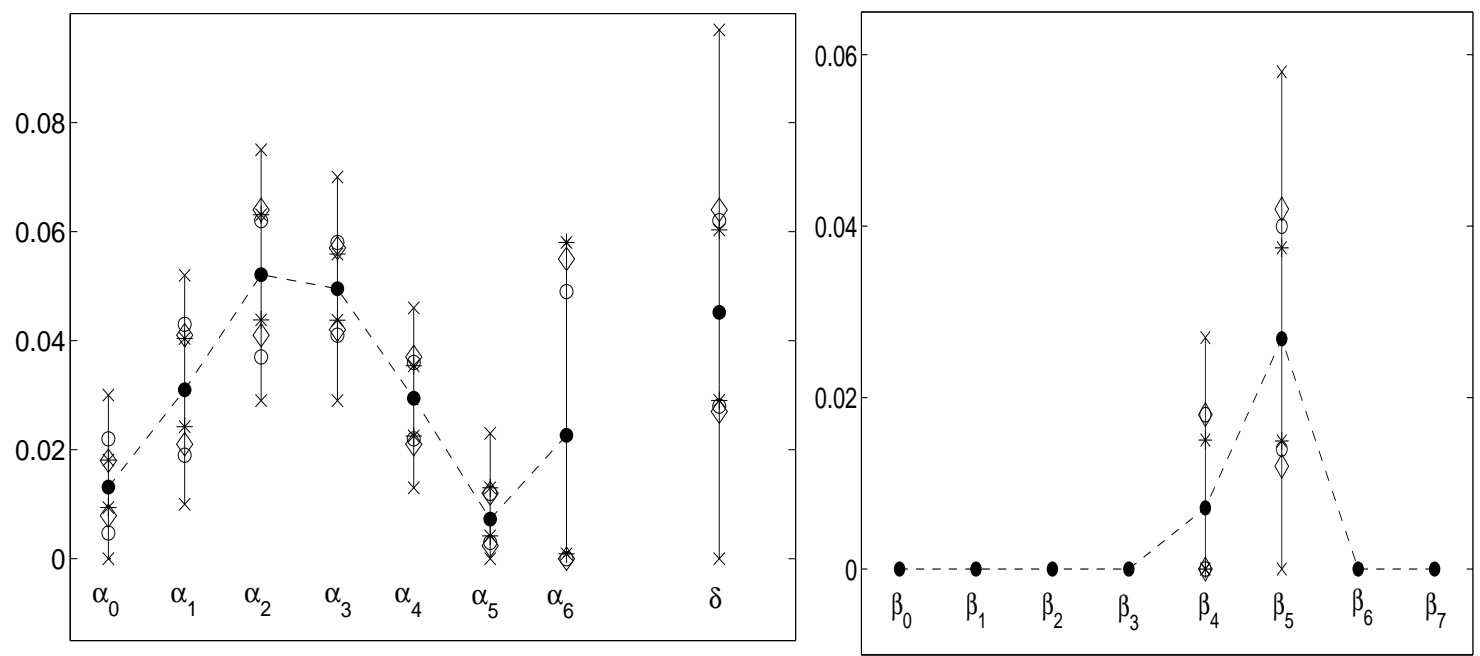

Figure 7: Comparison of the approximations to $95 \%$ confidence intervals for the best-fit parameter values of the heterogenous model computed by three methods: the variance-covariance method $(\diamond)$, the profile-likelihood-based method $(*)$ and two versions of the bootstrap method - non-parametric $(\times)$ and parametric (o) approaches. The best-fit parameter values are marked by $(\bullet)$.
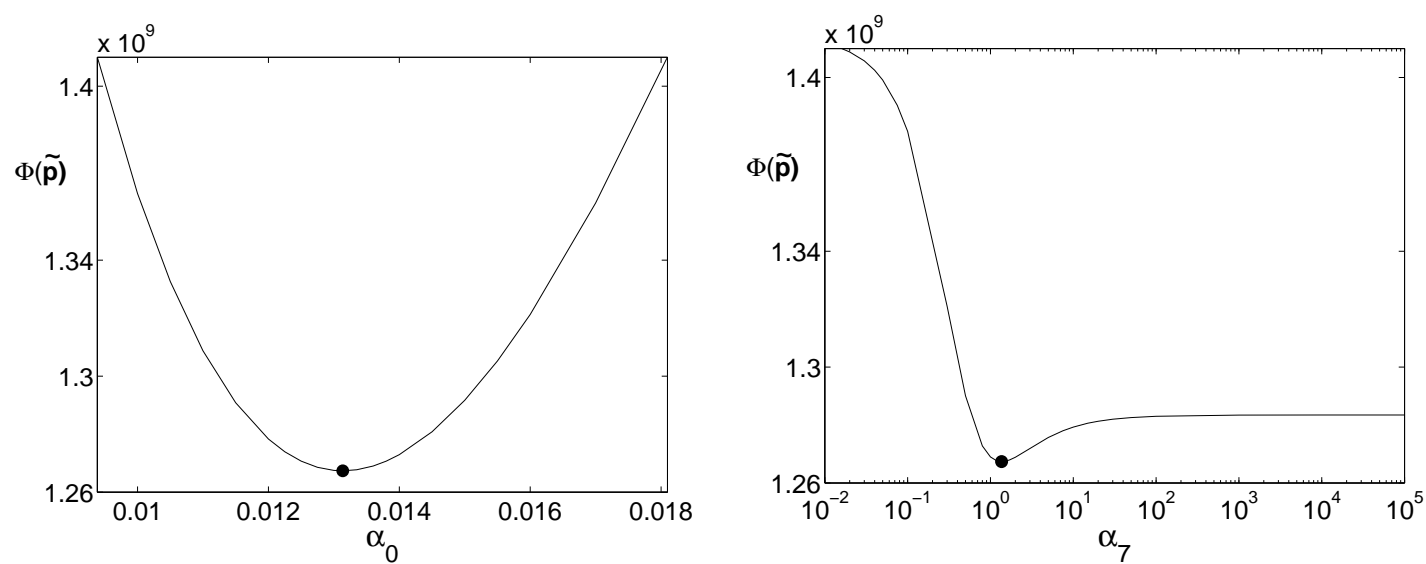

Figure 8: The behavior of the minimized least-squares function $\Phi(\tilde{\mathbf{p}})$ with respect to the naive cell division rate $\alpha_{0}$ (left) and with respect to the division rate of cells which have undergone 7 divisions, $\alpha_{7}$ (right). The parameters $\alpha_{0}$ and $\alpha_{7}$ vary within the computed $95 \%$ confidence interval, respectively, the interval $\left[\alpha_{7}^{\min }, 10^{5}\right]$. The profile of $\Phi(\tilde{\mathbf{p}})$ sheds light on the difficulty with computing the upper limit of the confidence interval for $\alpha_{7}$. The best-fit values of $\alpha_{0}$ and $\alpha_{7}$ are denoted by $(\bullet)$. 

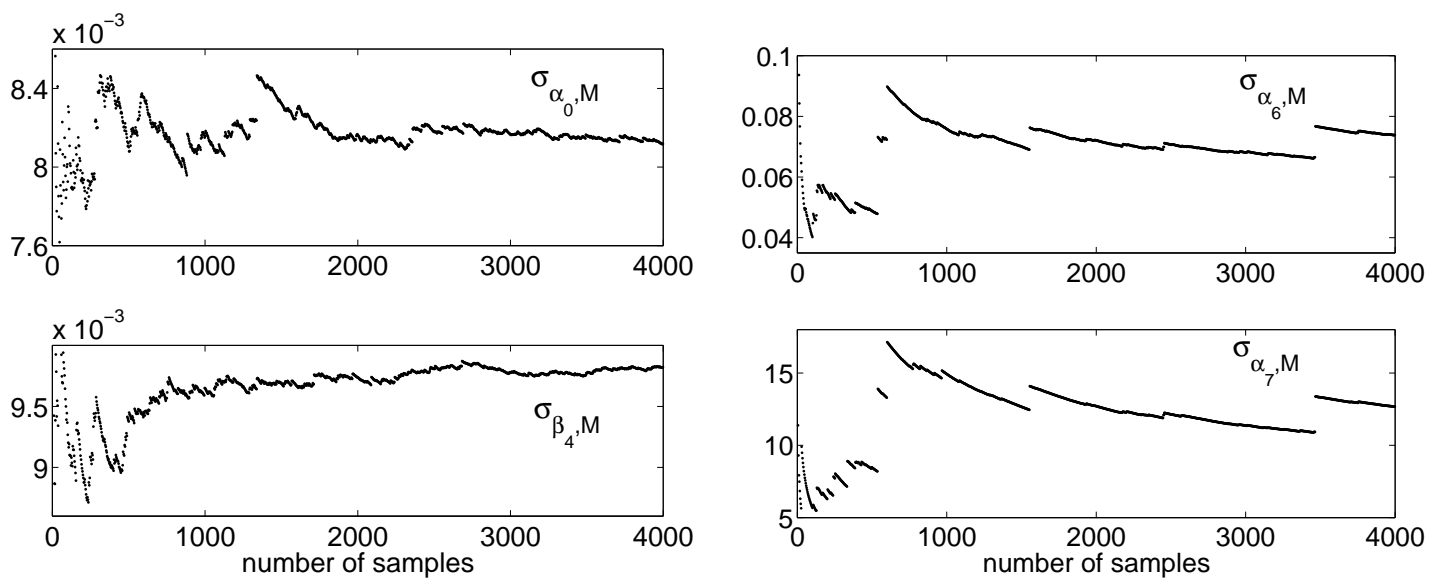

Figure 9: Evolution of the bootstrap estimates for the standard deviations $\sigma_{\alpha_{0}, M}, \sigma_{\beta_{4}, M}$ (left) and $\sigma_{\alpha_{6}, M}, \sigma_{\alpha_{7}, M}$ (right) versus the number of resamples $M$.
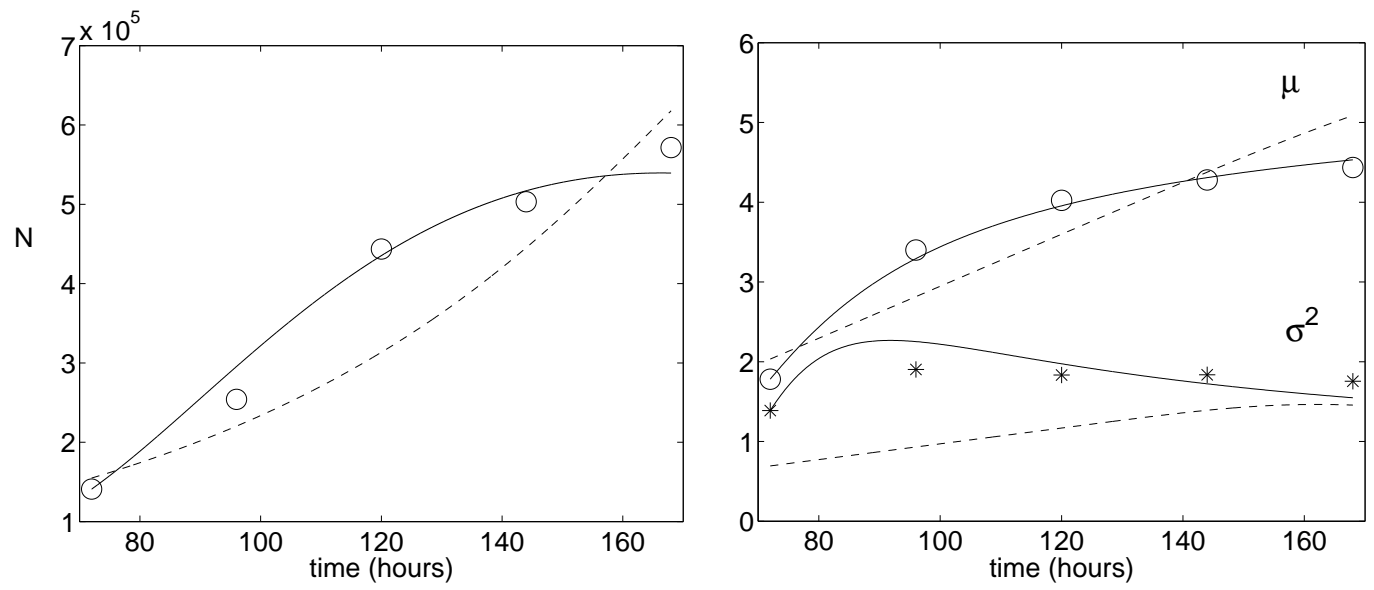

Figure 10: Performance of the heterogenous Kendall type model (3.1) and the homogenous SmithMartin model (6.1) on analysis of the in vitro data in Table 1. Left: The total number of live lymphocytes $N$ over all divisions: data (o) and the best-fit approximations provided by the heterogenous model (solid line) and the Smith-Martin model (dashed line). Right: The behavior of the mean division number $\mu(\circ)$ and its variance $\sigma^{2}(*)$ suggested by the data. The solid and dashed curves correspond to $\mu(t)$ and $\sigma^{2}(t)$ computed using the best-fit solutions of the heterogeneous and Smith-Martin models, respectively. 

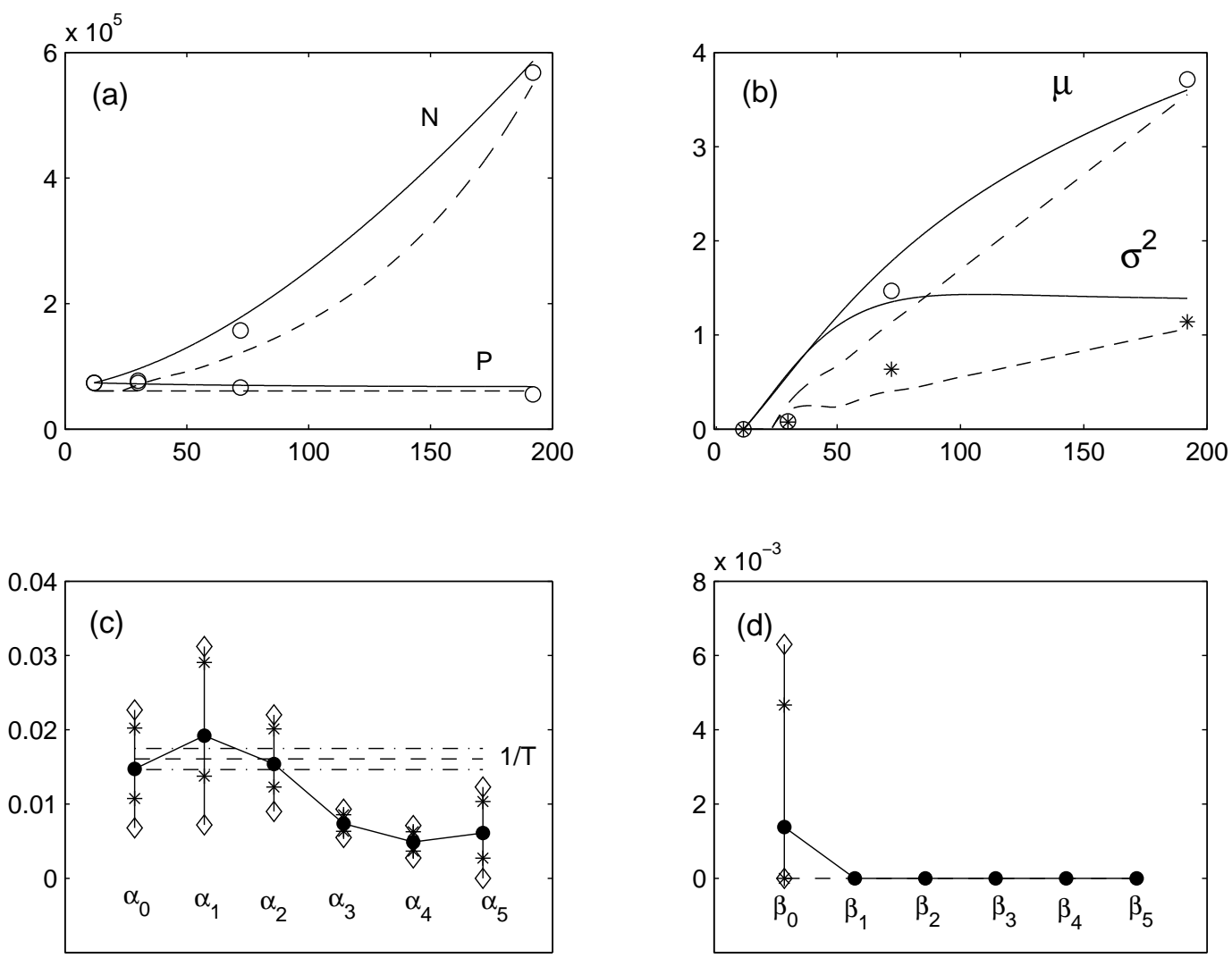

Figure 11: Analysis of in vivo mouse data on transgenic CD8 T cell growth following adoptive transfer into irradiated hosts given in [9]. The performance of the heterogenous model (3.1) and the homogenous Smith-Martin model (6.1) is analyzed. (a) The kinetics of the total number of live lymphocytes $N$ over all divisions and the number of precursors $P$ that would have generated the current lymphocyte population in the absence of death: estimated from the data (o) and predicted by the solutions of the heterogenous (solid curves) and Smith-Martin (dashed curves) models with the best-fit parameters. (b) The behavior of the mean division number $\mu(\circ)$ and its variance $\sigma^{2}(*)$ suggested by the data. The solid and dashed curves correspond to $\mu(t)$ and $\sigma^{2}(t)$ computed using the best-fit solutions of the heterogeneous and Smith-Martin models, respectively. (c)-(d) The best-fit parameter estimates of the heterogenous model $(\bullet)$ and the best-fit values of parameters $1 / T, d_{A}$ and $d_{B}$ of the Smith-Martin model (indicated by dashed curves, $d_{A} \approx d_{B} \approx 0$ ). Approximations to $95 \%$ confidence intervals for the best-fit parameters of the heterogeneous model computed by the variance-covariance method $(\diamond)$ and by the profile-likelihood-based method $(*)$ are shown. For the Smith-Martin model, the $95 \%$ confidence intervals for $1 / T$ computed by these two methods agree closely (dot-dashed curves). 